\title{
A New Dynamic Path Planning Approach for Unmanned Aerial Vehicles
}

\author{
Chenxi Huang $\mathbb{D}^{1},{ }^{1}$ Yisha Lan, ${ }^{1}$ Yuchen Liu, ${ }^{1}$ Wen Zhou $\mathbb{D}^{2}{ }^{2}$ Hongbin Pei, ${ }^{1}$ Longzhi Yang, ${ }^{3}$ \\ Yongqiang Cheng $\mathbb{D}^{4},{ }^{4}$ Yongtao Hao $\mathbb{D}^{1},{ }^{1}$ and Yonghong Peng $\mathbb{D}^{5}$ \\ ${ }^{1}$ Department of Computer Science and Technology, Tongji University, Shanghai 201804, China \\ ${ }^{2}$ School of Software Engineering, Tongji University, Shanghai 200065, China \\ ${ }^{3}$ Department of Computer and Information Sciences, Northumbria University, Newcastle upon Tyne NE1 8ST, UK \\ ${ }^{4}$ School of Engineering and Computer Science, University of Hull, Hull HU6 7RX, UK \\ ${ }^{5}$ Faculty of Computer Science, University of Sunderland, St Peter Campus, SR6 ODD, UK
}

Correspondence should be addressed to Yongtao Hao; hao0yt@163.com and Yonghong Peng; Yonghong.Peng@sunderland.ac.uk

Received 1 August 2018; Accepted 21 October 2018; Published 5 November 2018

Guest Editor: Junpei Zhong

Copyright (c) 2018 Chenxi Huang et al. This is an open access article distributed under the Creative Commons Attribution License, which permits unrestricted use, distribution, and reproduction in any medium, provided the original work is properly cited.

\begin{abstract}
Dynamic path planning is one of the key procedures for unmanned aerial vehicles (UAV) to successfully fulfill the diversified missions. In this paper, we propose a new algorithm for path planning based on ant colony optimization (ACO) and artificial potential field. In the proposed algorithm, both dynamic threats and static obstacles are taken into account to generate an artificial field representing the environment for collision free path planning. To enhance the path searching efficiency, a coordinate transformation is applied to move the origin of the map to the starting point of the path and in line with the source-destination direction. Cost functions are established to represent the dynamically changing threats, and the cost value is considered as a scalar value of mobile threats which are vectors actually. In the process of searching for an optimal moving direction for UAV, the cost values of path, mobile threats, and total cost are optimized using ant optimization algorithm. The experimental results demonstrated the performance of the new proposed algorithm, which showed that a smoother planning path with the lowest cost for UAVs can be obtained through our algorithm.
\end{abstract}

\section{Introduction}

Unmanned aerial vehicle (UAV) is a type of aircraft that can be remotely controlled or fly autonomously following on preplanned flying routes with no onboard pilots. UAV has found increasing number of applications in real-world environments $[1,2]$. With the incessant improvement of the maneuverability, stealth, aggression, and intelligence of the UAV, the application of UAV has been greatly expanded during the past years. The tasks for UAV to perform are diversified, such as reconnaissance and early-warning, electronic countermeasure, communication relay, and air striking [3-5]. Dynamic path planning is the key function of UAV to ensure that UAVs can carry out the tasks successfully. It mainly refers to plan an optimal flight track for UAV from a starting point to a target point which satisfies the constraints of the UAV's own performance expectation and the environment.
Furthermore, an optimized path guarantees the successful completion of the mission with the minimized cost [6-8].

Based on the mastery degree of the surrounding information, path planning can be constructed by two planning engines including a global path planning engine that uses prior environmental information for global planning and a local path planning engine that responds to the real-time sensor information, such as size and location of an obstacle. The local path planning is suitable for the situation where a starting point and a destination are relatively close. The local path planning is quick, real-time, and responsive, but it is easily distracted by local information thus falling into a local optimal solution without achieving the global target. Global path planning provides solution for overall path finding which finds out a both feasible and optimal path based on the environmental information obtained. All the environmental information is acquired firstly and then a preliminary (global) 
path planning is carried out according to the environmental map constructed. The global path planning relies on global environmental information and cannot deal with real-time problems during planning process. Global path planning demands large computational capacity. Meanwhile, local path planning is a kind of dynamic programming while global path planning belongs to static programming $[9,10]$.

Considerable literatures have been reported on the subject of path planning. The main methods currently developed for path planning include graph-based, populationbased evolutionary algorithms, and distributed approaches [11]. The graph-based algorithms include Voronoi diagram search method, directed graph-based method, $A *$ search algorithms, mathematical programming method, artificial potential field method (APF), and $\mathrm{D} *$ lite algorithms. The graph-based algorithms, however, have shown weakness in practical situations due to its difficulty to address the motion constraints of UAV. The population-based algorithms contain particle swarm optimization (PSO), ant colony optimization (ACO), intelligent water drops optimization (IWD), gravitational search algorithm (GSA), and artificial bee colony $(A B C)$. The population-based evolutionary algorithms have advantages of reduced complexity and dimensions in the planning phase $[12,13]$. Distributed path planning off loads the path planning to environment using message exchanges between intelligent nodes. While these methods require minimized onboard processing, they rely on the smart infrastructures to be preinstalled. All of these methods have been applied to path planning in the literature, but each of them has its own disadvantages in certain aspects. For example, the APF method fails to generate path effectively in the situation with a more dense group of obstacles. The target cannot be reached if there are obstacles near the target. Moreover, jitter may occur in the planning path when it is near to an obstacle or in a narrow passageway $[14,15]$. As for the ACO algorithm, it requests large amount of computation and the efficiency very much depends on the parameter. If the parameter is set incorrectly, the solution speed is slow and the quality of the solution is poor. Although an optimal solution can be obtained through exchanging information, the efficiency will be inevitably reduced when the content of the problem is too large. Meanwhile, ACO algorithm has a slow convergence speed and can easily fall into local optimal solution due to its lack of initial information [16, 17].

To address the above challenges, we propose a new model for UAV path planning based on the ant colony optimization algorithm and the artificial potential field algorithm. Both mobile threats and static threats are considered with a set influence radius. Furthermore, mobile threats such as radars, missiles, artillery, and atmosphere are given with their moving directions. During the process of initialization, an occupant grid map will be constructed under the transformed coordinate system using the environmental information available. The initial coordinates of the points on the paths will be set. Then the optimum moving directions are searched based on probability and the cost of mobile threats from the starting point. The pheromone and next step of movement are updated iteratively until the target is reached with the best solution.
We evaluate the performance of the proposed method in planning paths against the performance of ACO algorithm in two experiment scenarios: (1) with both mobile threats and static threats presented; (2) with only mobile threats. The experimental results show that our proposed algorithm has lower costs and achieves higher performance producing a smoother path. The costs include the cost of mobile threats, the cost of path, and the total cost, while the total cost is the sum of the cost of mobile threats and the cost of path. And the cost of path is mainly considered as the fuel consumption in the entire path.

The contributions of the paper can be summarized as follows: both mobile threats and static threats are considered to simulate the actual situations better and a coordinate system transformation is used for simplicity. Meanwhile, in ACO algorithm, the thoughts of APF are used where the cost value is considered as a scalar value of mobile threats which are vectors actually.

The remaining part of the paper is organized as follows. Related work is introduced in Section 2. The new UAV path planning model and algorithm are proposed in Section 3. The experimental results are presented in Section 4. Section 5 concludes the paper with future work.

\section{Related Work}

2.1. Artificial Neural Network. Artificial Neural Network (ANN) is a method that mimics the neural network of organisms. It is a mathematical model established by abstracting the structure of the biological neural network and the mechanism responding to the outside stimulus. Nowadays, ANN has developed a multitude of models, such as Hopfield network, ART network, and Kohonen network [18]. It is a typical parallel computing model consisting of many interconnected simple processors which are also called neurons or nodes. There are only two rules for its computing capability. The former is the rule of combining signals input, which usually represents the weighted sum of the signals input, and the latter is the activation function that calculates the combined input signals into the output signals. Each processor only receives signals periodically and sends signals to other processors.

The ANN model is characterized by parallel distributed processing ability, high fault tolerance, and self-learning ability which can combine information processing and storage. ANN has excellent performance in the task of dealing with parallel complex systems, so it is often used to solve problems with complex and mission critical applications [19].

2.2. Particle Swarm Optimization. Particle swarm optimization (PSO) originally proposed by Eberhart and Kennedy in 1995 is an intelligent search algorithm that mimics the foraging behavior of birds. In this algorithm, every potential solution of the problem to be optimized is represented as a point in the $\mathrm{N}$ dimensional space which is called particle. Each particle has a fitness function determined by fitness function and a speed determining the direction and speed of a particle. Particles move at the speed of their own towards the direction of the currently most optimal particle. The 


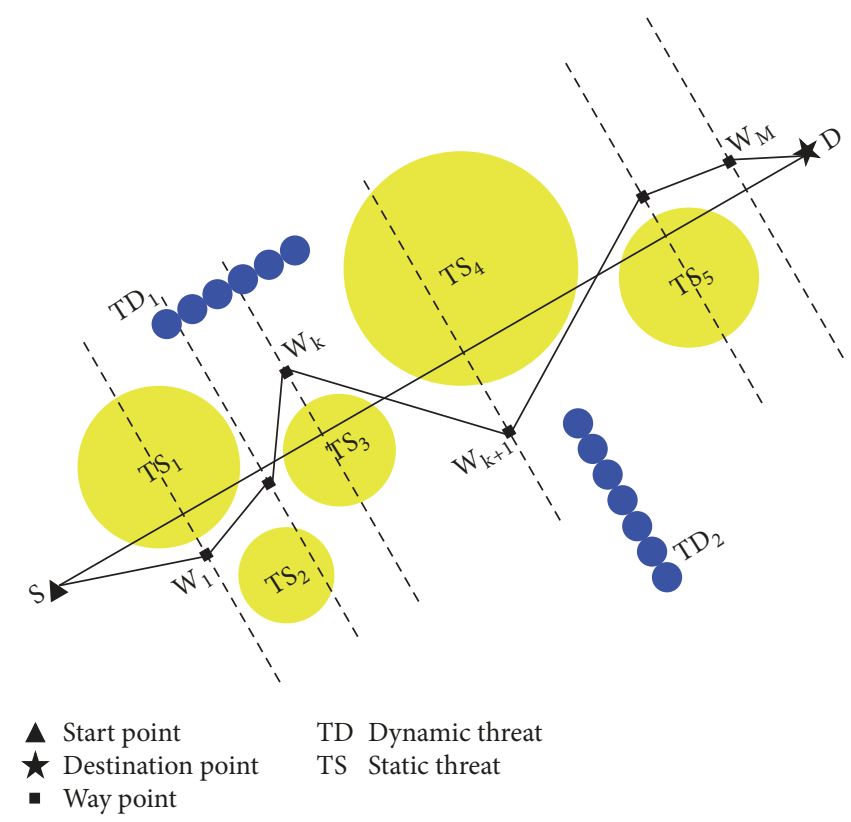

(a)

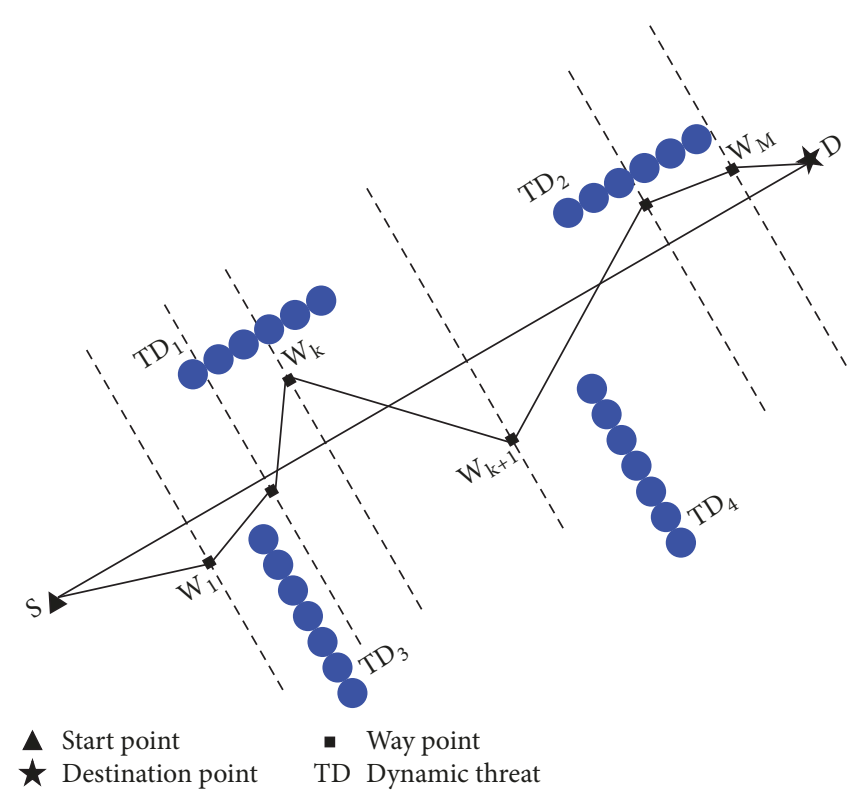

(b)

FIGURE 1: Typical UAV path planning model. (a) contains both mobile threats and static threats while (b) only contains the mobile threats.

PSO is widely adopted for robot path planning because it requires only small computation resources, has fast search speed, and uses less tuning parameters. A PSO algorithm is based on guaranteed convergence and applying it to the global path planning of mobile robots was proposed. The method is described as encoding the path which connects the start point and destination in the environment into particles. Then an active region of particles is divided according to the positions of obstacles, and the initial population is produced in this area, which makes a particle search an optimal path in a smaller restricted area. In the search process, the acceleration coefficient and inertia weight of particles are adjusted adaptively with the increase of iterations [20-22].

2.3. Genetic Algorithm. Genetic algorithm (GA) is first proposed by Professor J. Holland in the United States in 1975 which is a kind of intelligent search algorithm which simulates the process of biological evolution to find an optimal solution. GA is started from a randomly generated or specific initial population which consists of several genetically encoded individuals. In GA, certain common operations, such as selection, replication, cross, and variation are used. According to the fitness of an individual and the principle of survival of the fittest, a new generation of population is produced through incessant iterative calculation. In this way, an optimal solution or an approximate optimal solution is obtained after decoding the genes of the optimal individuals. Because of inherent parallelism and great ability of global optimization of GA, genetic algorithm is widely used in function optimization, machine learning, signal processing, adaptive control, and robot path planning.

In view of the problem of many factors involved in the path of 3D terrain, a path planning method based on multiobjective genetic algorithm was put forward. In this algorithm, the total length and slope of the path and the path security are taken into account to measure the path obtained. In order to avoid the loop in the path, the initial population is obtained by using large scale uniform initialization, and loops of the iteration are then removed through the introduction of the austerity operator $[23,24]$.

2.4. Sparse A* Search. Sparse A* Search (SAS) algorithm is an improved $A *$ algorithm proposed by Szczerba et al. in 2000. SAS combines the constraints of the problems to be solved in the search process of the basic $A *$ algorithm to reduce the number of unused nodes in the search space, thus reducing the time consumed by the algorithm. These constraints are the maximum corner constraint and the maximum path length, and a method based on a new structured search space was proposed. That is to say, in a planning area, a sufficient number of nodes are generated uniformly by a random function, and the nodes are validated through visibility to form a search space. This method can significantly reduce the computational complexity and improve the search efficiency. Moreover, it can also flexibly balance between the path quality and time consumption $[25,26]$. SAS is currently widely used in path planning of 3D environment.

\section{The Proposed Method}

3.1. Model Building. UAV path planning is a global optimization problem to generate a set of way points from the start point $S$ to the destination point $\mathrm{D}$ with guarantee not to fly out of the designated map or being taken by threats. In our work, all threats located in the environment are divided into two categories, i.e., mobile threats and static threats. As shown in Figure 1, static threats are obstacles with a static position in the space (denoted as yellow circles with 


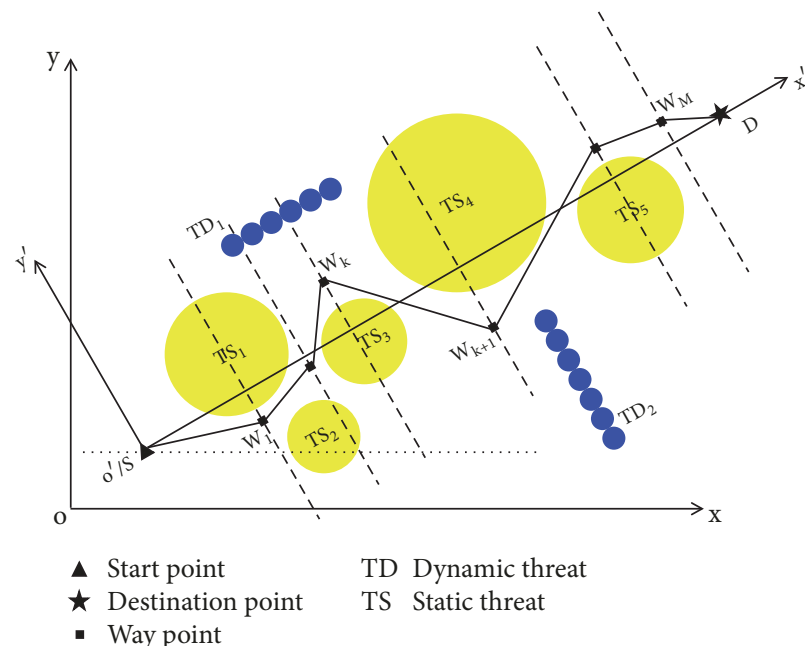

(a)

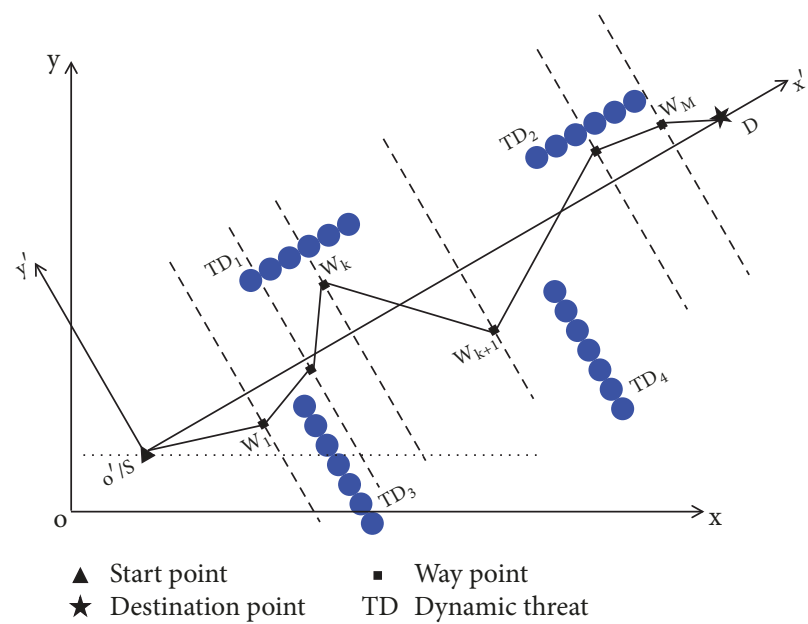

(b)

FIGURE 2: Transformation of coordinate system in UAV path planning model. (a) contains both mobile threats and static threats while (b) only contains mobile threats.

set radius); mobile threats are dynamically moving obstacles with a known constant velocity (denoted as blue circles). In Figure 1, the straight line $\mathrm{SD}$ is divided into $(\mathrm{M}+1)$ segments, and $W_{k}(k=1,2, \cdots, M)$ denote the $M$ way points along the flying route.

As shown in Figure 2, a transformation of coordinate system is produced to reduce the complexity of computation process in the UAV path planning. By setting the straight line between the $\mathrm{S}$ and $\mathrm{D} \overrightarrow{S D}$ as the new $\mathrm{x}$-axis and the start point (S) of UAV as the new origin of the coordinate, a new coordinate system $x^{\prime} o^{\prime} y^{\prime}$ is then transformed from the original coordinate system $x o y$. If a way point in xoy system and $x^{\prime} o^{\prime} y^{\prime}$ system is given as $\left(x_{o}, y_{o}\right)^{T}$ and $\left(x_{t}, y_{t}\right)^{T}$; the relationship between the original and transformed coordinates in the two coordinate systems can be expressed as follows:

$$
\left[\begin{array}{l}
x_{t} \\
y_{t}
\end{array}\right]=\left[\begin{array}{cc}
\cos \theta & \sin \theta \\
-\sin \theta & \cos \theta
\end{array}\right]\left[\begin{array}{l}
x_{o}-x_{s} \\
y_{o}-y_{s}
\end{array}\right]
$$

where $\left(x_{s}, y_{s}\right)^{T}$ is the coordinate of start point in the original coordinate system.

And the cost of mobile threats is mathematically given as

$$
\begin{aligned}
|\vec{c}| & =\frac{1}{d_{i j}^{2}} \\
\left|\overrightarrow{c_{x}}\right| & =|\vec{c}| \cdot \frac{x_{i j}}{d_{i j}}=\frac{x_{i j}}{d_{i j}^{3}} \\
\left|\overrightarrow{c_{y}}\right| & =|\vec{c}| \cdot \frac{y_{i j}}{d_{i j}}=\frac{y_{i j}}{d_{i j}^{3}} \\
\left|\sum_{k=1}^{m} \vec{c}\right| & =\sqrt{\left|\sum_{k=1}^{m} \overrightarrow{c_{x}}\right|^{2}+\left|\sum_{k=1}^{m} \overrightarrow{c_{y}}\right|^{2}}
\end{aligned}
$$

where $|\vec{c}|$ is a mobile threat and $\left|\overrightarrow{c_{x}}\right|$ and $\left|\overrightarrow{c_{y}}\right|$ are, respectively, the horizontal component and vertical component of $|\vec{c}|$. And $d_{i j}$ is the distance between positions $\mathrm{i}$ and $\mathrm{j}, x_{i j}$ is value of the horizontal component of $d_{i j}$, and $y_{i j}$ is the value of the vertical component of $d_{i j}$. Moreover, $\mathrm{m}$ is the number of mobile threats affecting the ant. Finally, positions of each ant and pheromone are updated in time.

The performance indicators of the planned path mainly consist of the mobile threat cost $I_{T}$, the fuel cost $I_{F}$, and the total cost $I$. The calculation formulas of them are expressed as follows:

$$
\begin{gathered}
I_{T}=\sum_{i=0}^{M} I_{T}(i) \\
I_{F}=\sum_{i=0}^{M} I_{F}(i) \\
I=I_{T}+I_{F}
\end{gathered}
$$

where $I_{T}(i)$ and $I_{F}(i)$ denote the mobile threat cost and the fuel cost at the ith subpath from $W_{i}$ to $W_{i+1}$, respectively. The mobile threat cost of subpath is calculated by an approximation based on blue circles along the subpath as shown in Figure 1. If the ith subpath $\left(W_{i}, W_{i+1}\right)$ is within the effect range, the threat cost is given as

$$
I_{T}(i)=L_{i} \sum_{k=1}^{N_{t}} T_{k} \cdot c_{k}
$$

where $N_{t}$ denotes the number of mobile threats, $L_{i}$ represents the length of ith subpath, and $T_{k}$ denotes the weight of each mobile threat. And $c_{k}$ is the cost of each mobile threat which is calculated by (5). Meanwhile, if $w_{F}$ represents the weight of fuel, then the fuel cost of the ith subpath is given as

$$
I_{F}(i)=w_{F} \cdot L_{i}
$$




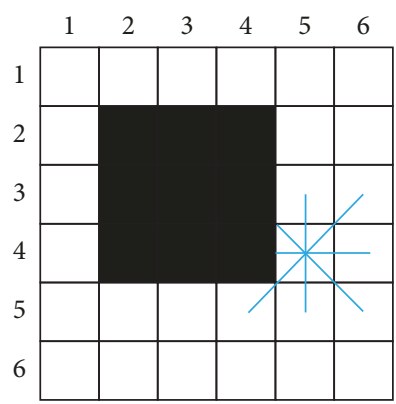

FIGURE 3: Schematic diagram of the ant colony optimization algorithm search process.

3.2. Ant Colony Optimization Algorithm. The basic mathematical model of ant colony algorithm was firstly applied to the traveling salesman problem. The problem can be described as finding the shortest path for a businessman to visit all cities at least and at most once. Let us assume there are $\mathrm{m}$ cities and $\mathrm{N}$ salesmen, where each ant chooses the next city to move to at an identical distance and releases the consistent pheromone. In the grids, each ant can only choose one grid which is adjacent to the grid it locates now. As shown in Figure 3, when an ant is in grid $(4,5)$, all the grids it can choose from are $(3,4),(3,5),(3,6),(4,4),(4,6),(5,4),(5,5)$, and $(5,6)$. Because grids $(3,4)$ and $(4,4)$ are occupied by obstacles, these two grids will be ignored by ants.

The probability of ant $t$ moving from location i to location $\mathrm{j}$ is given as

$$
\begin{aligned}
& P_{i j}^{t}(x) \\
& = \begin{cases}\frac{\left[\tau_{i j}(x)\right]^{\alpha}\left(\eta_{i j}\right)^{\beta}}{\sum_{t \in \text { allowed } t(x)}\left[\tau_{i j}(x)\right]^{\alpha}\left(\eta_{i j}\right)^{\beta}}, & \text { if } j \in \text { allowed } t(x) \\
0, & \text { otherwise }\end{cases}
\end{aligned}
$$

where $\alpha$ is a heuristic factor of pheromone concentration and $\beta$ is also a heuristic factor of expectation which is the basis for ants to choose next location. An ant tends to choose the path that has been chosen by most ants with the increase of $\alpha$ and also choose the grid which is closer to the destination with the increase of $\beta$. And $\eta_{i j}$ represents a heuristic function of expectation defined by the equation

$$
\eta_{i j}=\frac{1}{d_{i j}}
$$

where $d_{i j}$ is the distance between locations $\mathrm{i}$ and $\mathrm{j}$. And allowed $t(x)$ is a set of next nodes which are allowed for ants to choose and it can be mathematically described as

$$
\text { allowed } t(x)=\{1,2, \cdots, n\}-\text { tab } u_{t}
$$

where $t a b u_{t}$ represents a tabu list to record the path of ant $t$.

Pheromone resides on the path of ants and it can volatile over time. Thus the equation of updating pheromone is expressed as follows.

$$
\tau_{i j}(x+m)=(1-\rho) \cdot \tau_{i j}(x)+\Delta \tau_{i j}
$$

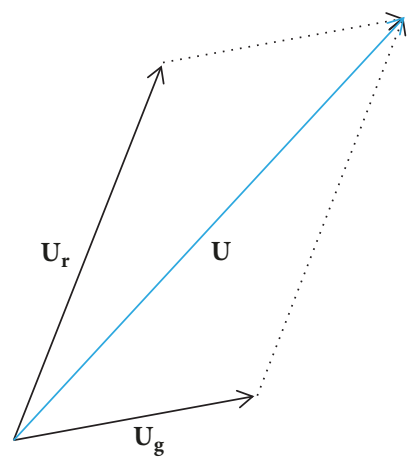

FIGURE 4: Schematic diagram of geometrical superposition principle.

where $\rho$ is a parameter and $\rho \in(0,1) . \Delta \tau_{i j}$ is described as

$$
\Delta \tau_{i j}= \begin{cases}\frac{Q}{L_{t}}, & (i, j) \in P_{t}(\text { begin, end }) \\ 0, & \text { otherwise }\end{cases}
$$

Q is the intensity of pheromone and $L_{t}$ is the path length of ant $t$ in a cycle. $P_{t}$ is the path taken by ant $t$ from the starting point to the destination in the cycle.

3.3. Artificial Potential Field Algorithm. Artificial potential field algorithm is based on repulsion or attraction forces applied by the object by simulating the gravity field in nature. In the potential field, an obstacle $\mathrm{O}$ has repulsion effect to the UAV A and varies in inverse proportion to the distance; that is, the closer the distance the greater the repulsion. Meanwhile, the destination D has attraction on A which is proportional to the distance, which means the attraction increases with the increase of the distance. As shown in Figure 4, A is affected by the virtual repulsion and attraction in an unknown environment, and the resultant force is the motive force of the UAV. The potential function is obtained by geometrical superposition principle.

$$
U=U_{r}+U_{a}
$$

where $\mathrm{U}$ is the total potential field, $U_{r}$ is the repulsive potential field, and $U_{a}$ is the gravitational potential field.

And the relationship of the resultant force $(\mathrm{F})$, repulsion force $\left(F_{r}\right)$, and attraction force $\left(F_{a}\right)$ are negative gradient of their potential fields given as follows.

$$
F=F_{r}+F_{a}
$$

Gravitational potential field function is defined by

$$
U_{a}(X)=\frac{1}{2} k\left(X-X_{d}\right)^{2}
$$

where $\mathrm{k}$ represents gravitational positive constant and $X_{d}$ and $X$ are the positions of destination and UAV, respectively. The direction of vector is located on the connection of the two positions and points to the destination. 


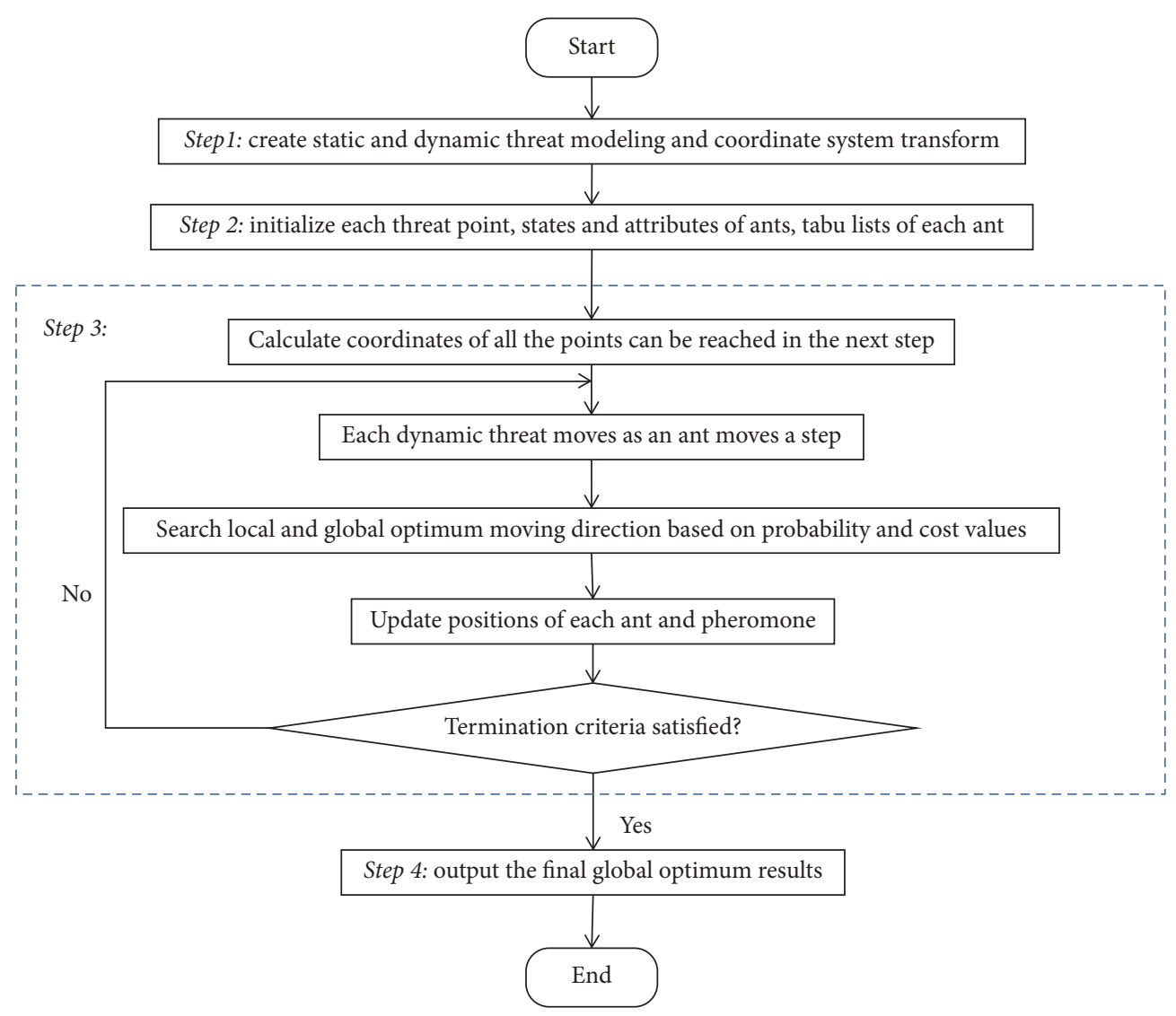

FIGURE 5: Flowchart of the proposed algorithm. follows.

Attraction $\left(F_{a}\right)$ is described as negative gradient given as

$$
F_{a}(X)=-\operatorname{grad}\left(U_{a}(X)\right)=k\left(X_{d}-X\right)
$$

In (10), when the destination $\mathrm{D}$ is farther away from the UAV A, both the gravitational potential energy and attraction to $\mathrm{A}$ are greater. When $\mathrm{A}$ is at the destination, the current gravitational potential energy is 0 .

Repulsive potential field function is defined as

$$
U_{r}(X)= \begin{cases}\frac{1}{2} m\left(\frac{1}{X-X_{o}}-\frac{1}{R}\right)^{2} & \left(X-X_{o}\right) \leq R \\ 0 & \left(X-X_{o}\right)>R\end{cases}
$$

where $m$ represents a positive constant of repulsive potential field, $\mathrm{R}$ is an influence scope of an obstacle, and $X_{o}$ is the position of an obstacle which cannot coincide with $X$. And when the distance between $\mathrm{O}$ and $\mathrm{A}$ is greater than $\mathrm{R}$, the repulsive potential field will no longer affect the path of UAV. The direction of vector is located on the connection of the obstacle and UAV and points to the UAV.
Repulsion $\left(F_{r}\right)$ which is described as negative gradient is given as follows.

$$
\begin{aligned}
& F_{r}(X)=-\operatorname{grad}\left(U_{r}(X)\right) \\
& = \begin{cases}m\left(\frac{1}{X-X_{o}}-\frac{1}{R}\right) \frac{1}{\left(X-X_{o}\right)^{2}} \cdot \frac{\partial\left(X-X_{o}\right)}{\partial X}, & \left(X-X_{o}\right) \leq R \\
0, & \left(X-X_{o}\right)>R\end{cases}
\end{aligned}
$$

In (12), when the destination $\mathrm{D}$ is farther away from the UAV A, both the gravitational potential energy and attraction to $\mathrm{A}$ are greater. When $\mathrm{A}$ is at the destination, the current gravitational potential energy is 0 . No repulsion function will be generated when $\mathrm{O}$ is out of the influence scope of $\mathrm{A}$. And when the distance between $\mathrm{O}$ and $\mathrm{A}$ is within the influence range of the repulsion field, the size of the repulsive potential field varies in inverse proportion to the distance.

3.4. The Proposed Algorithm. The proposed algorithm for the UAV path planning is presented in Figure 5 which combines ACO algorithm and APF algorithm.

Step 1 (path planning field formation). Firstly, a flying field for UAV is formulated which contains a starting point, a target point, and a rectangular region. Threats including mobile 


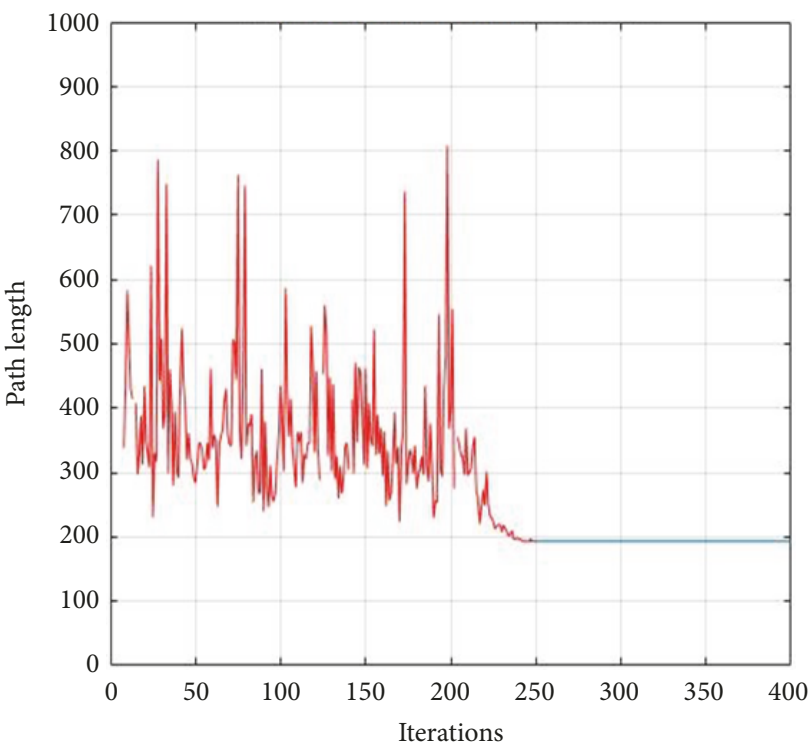

(a)

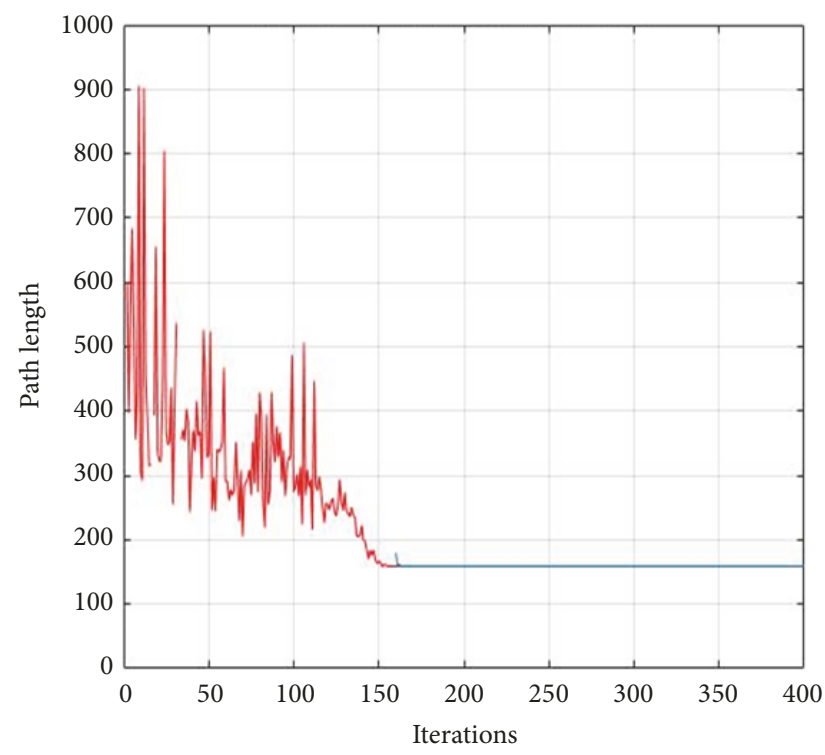

(b)

FIGURE 6: The optimal path length with the increase of iterations in one environment. There are both static and mobile threats in (a) and only mobile threats in (b).

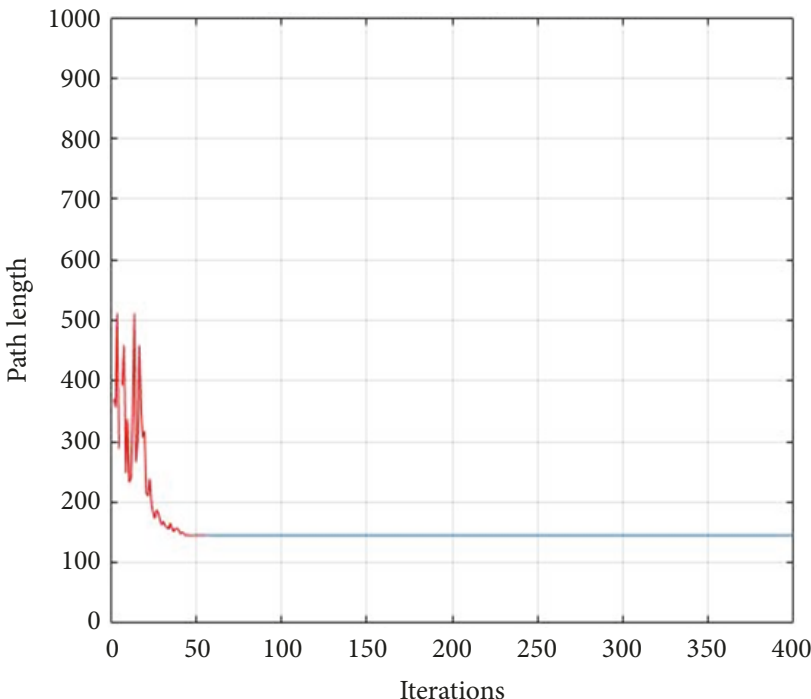

(a)

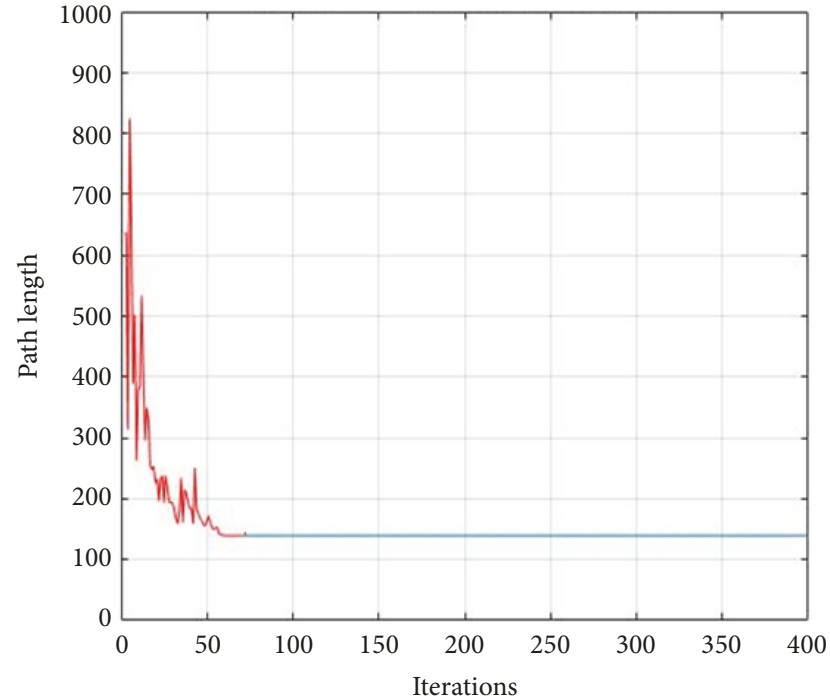

(b)

FIGURE 7: The optimal path length with the increase of iterations in another environment. There are both static and mobile threats in (a) and only mobile threats in (b).

threats and static threats are detected by UAV. The radius and position of static threats are known and the mobile threats and their moving direction will be sensed. All points coordinates will be converted into the new coordinate system using (1).

Step 2 (initialization). In the proposed algorithm, tabu lists of each ant and the states and attributes of ants are initialized. Moreover, the two-dimensional coordinates of range of motion for ants can be changed into one-dimensional array by compressing the vertical coordinate.
Step 3 (start algorithm iterations). The algorithm will iteratively update the positions and status once the initialization is finished until termination criteria are met. The termination criteria are set as an ant cannot reach the destination or fall into a dead circulation. Firstly, coordinates of all the points may be reached in the next step; that is, the points that are neither in the tabu list nor in the static threats are calculated. Then mobile threats start to move and each one moves a step as an ant moves a step. Next, the local and global optimum moving directions are searched based on probability in (6) and the cost of mobile threats. 


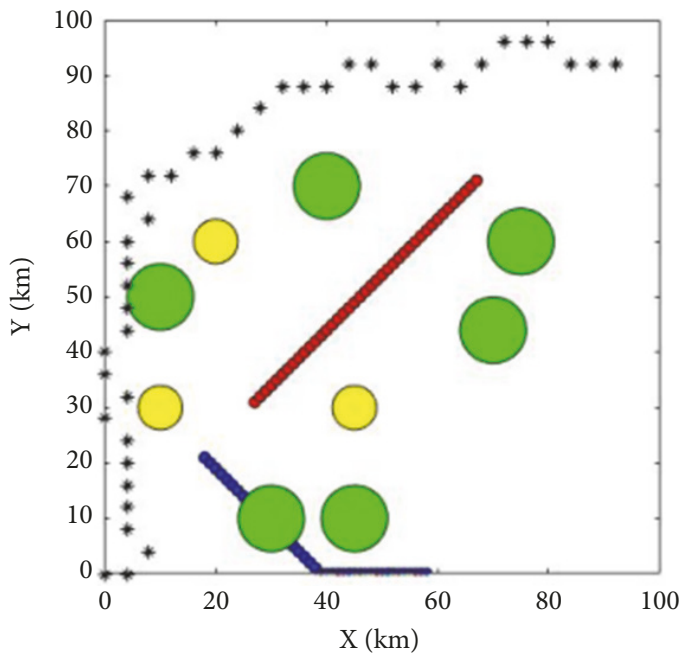

(a) Entire path

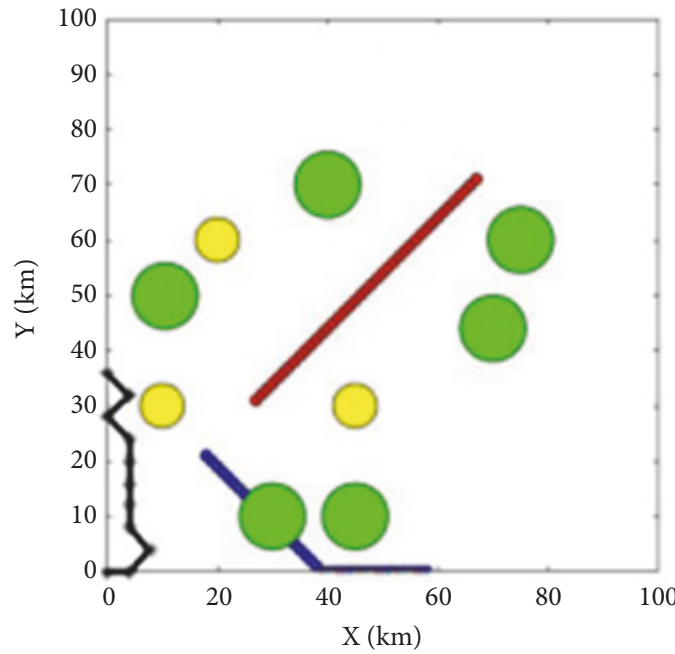

(b) $\mathrm{k}=100$

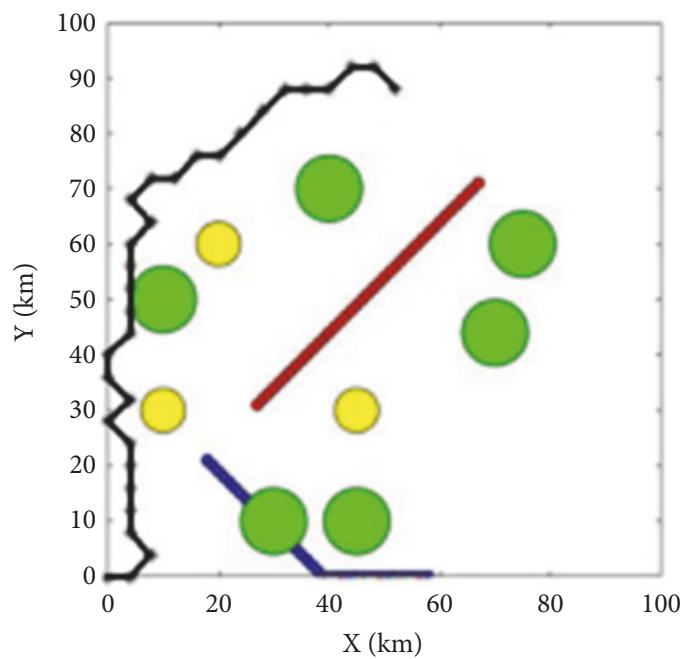

(d) $k=300$

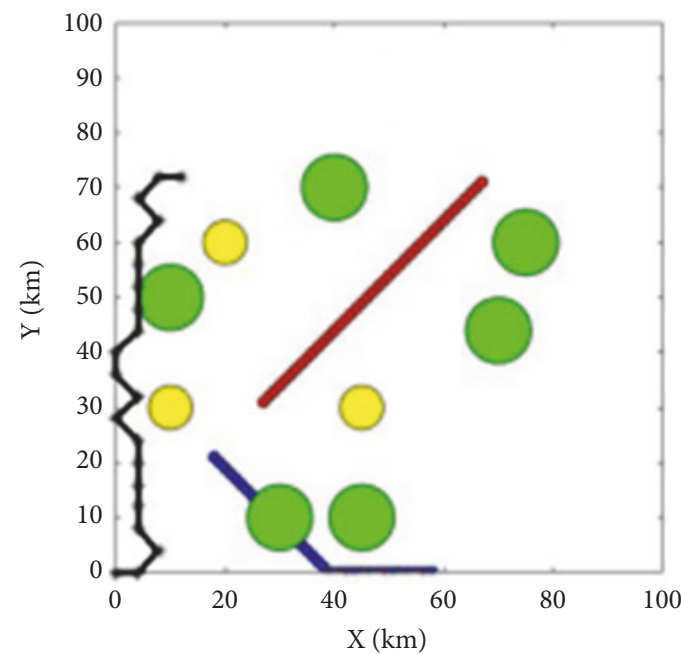

(c) $\mathrm{k}=200$

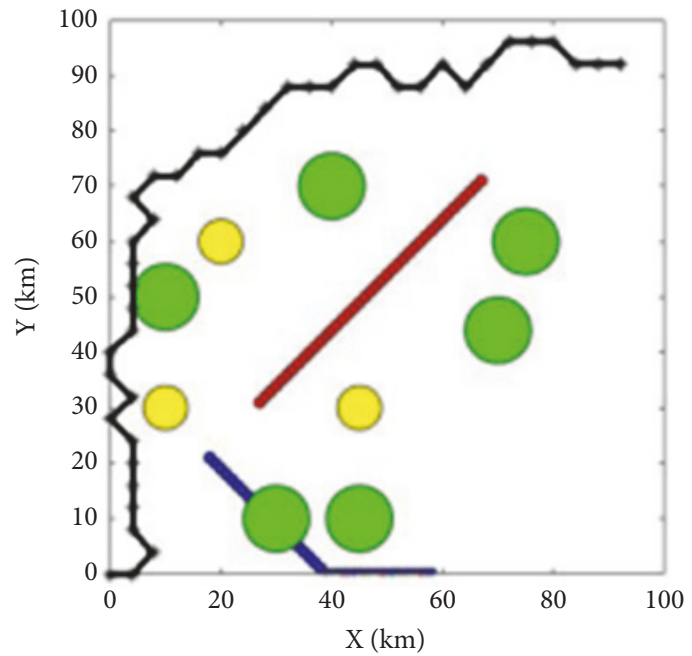

(e) $\mathrm{k}=400$

FIGURE 8: UAV path planning in one environment based on our proposed algorithm. And there are both static and mobile threats where (a) is an entire path and (b) to (e) are paths with iterations $=100,200,300,400$. 


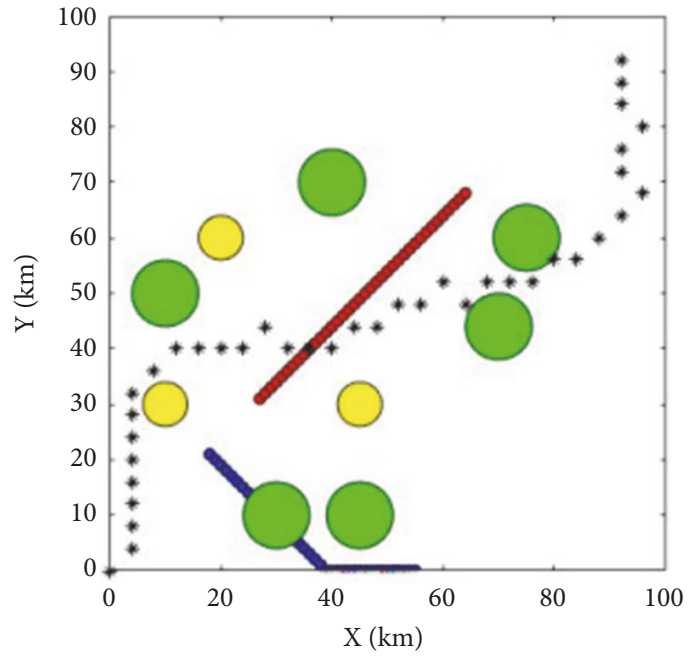

(a) Entire path

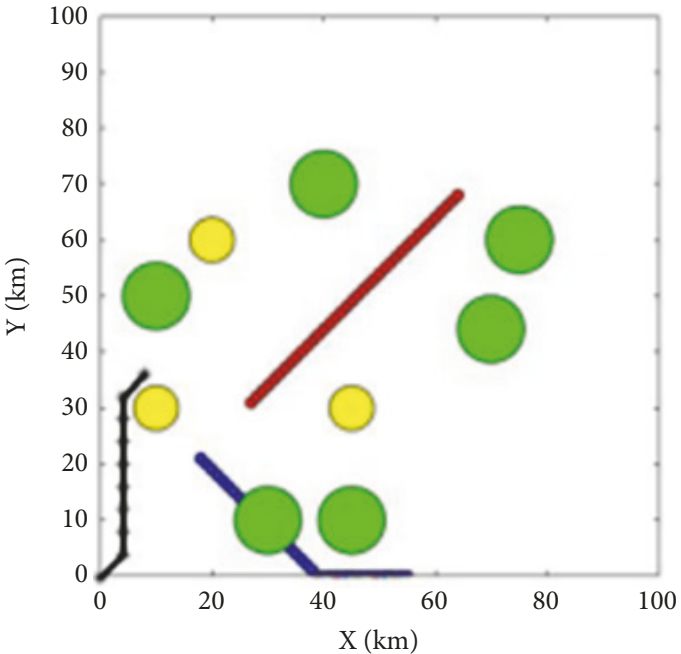

(b) $\mathrm{k}=100$

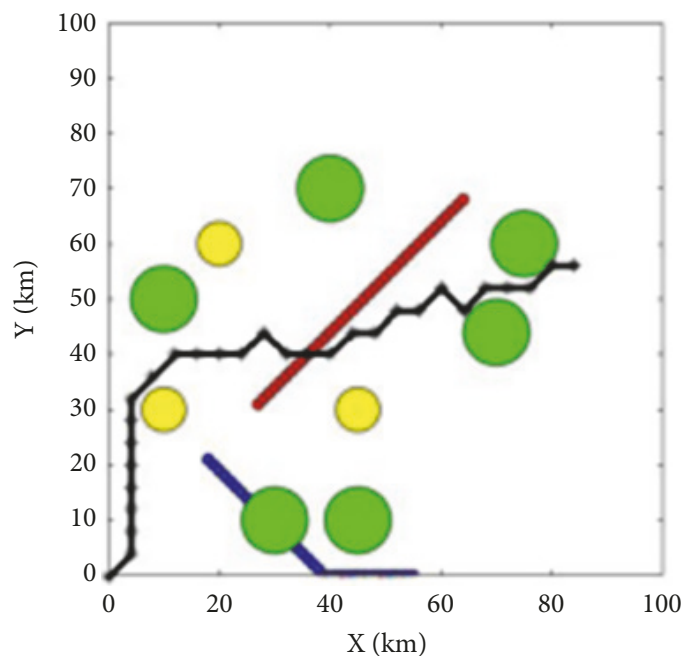

(d) $\mathrm{k}=300$

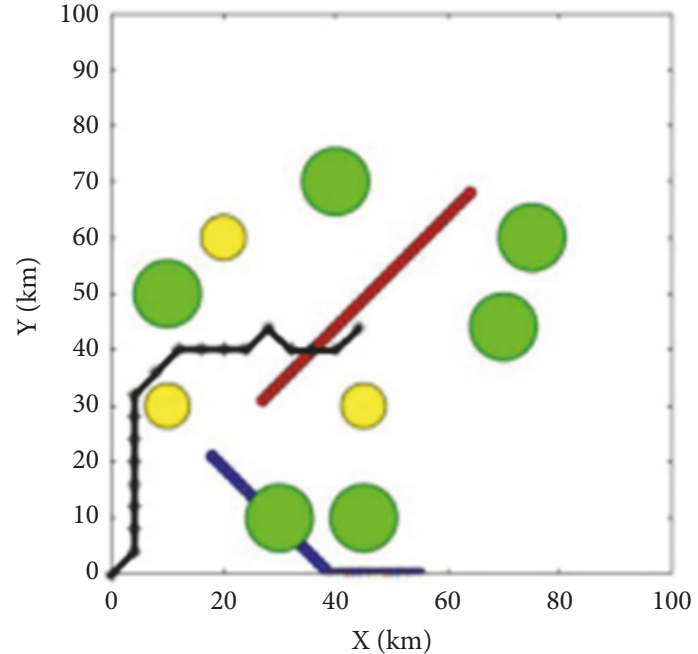

(c) $\mathrm{k}=200$

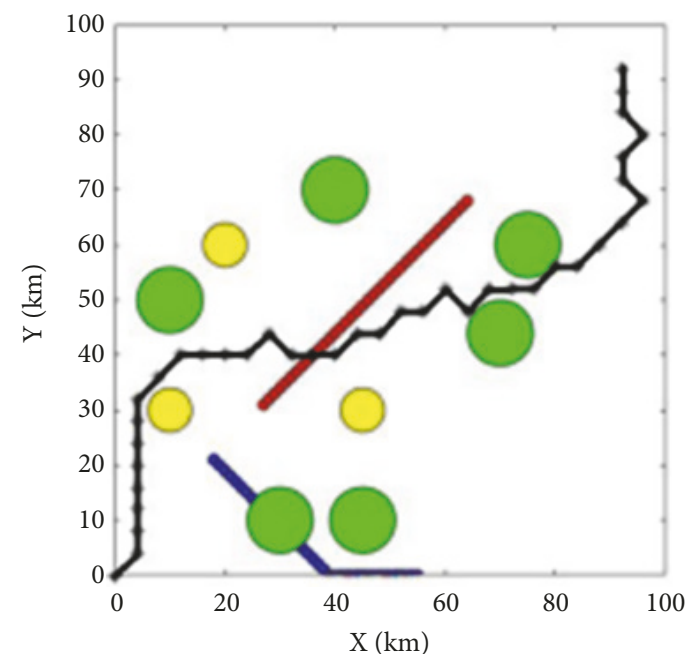

(e) $\mathrm{k}=400$

FIGURE 9: UAV path planning in one environment based on ACO algorithm. And there are both static and mobile threats where (a) is an entire path and (b) to (e) are paths with iterations $=100,200,300,400$. 


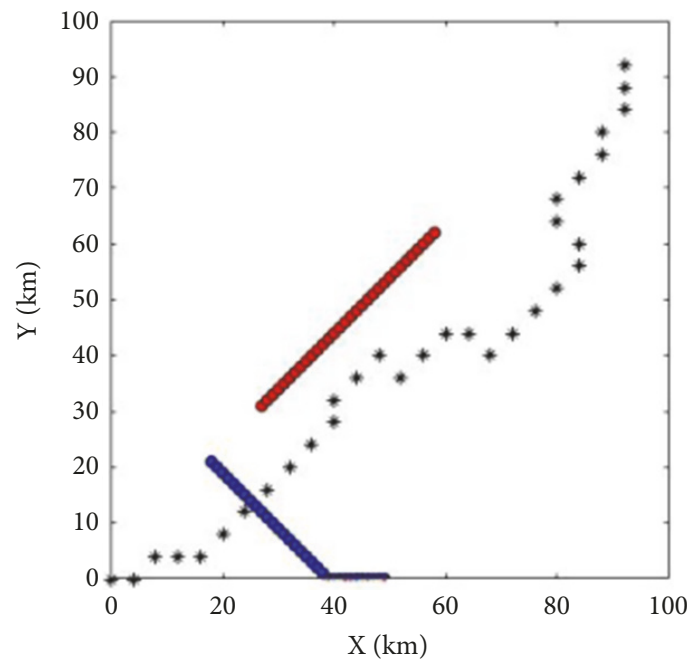

(a) Entire path

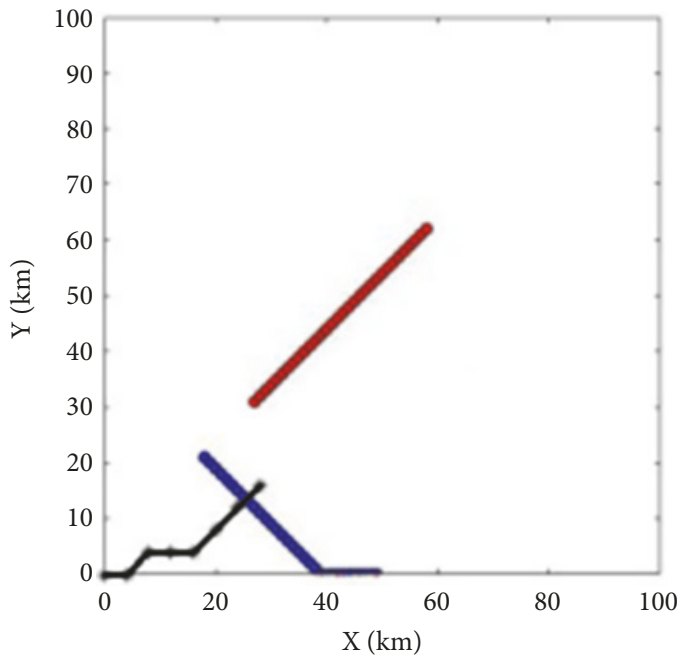

(b) $\mathrm{k}=100$

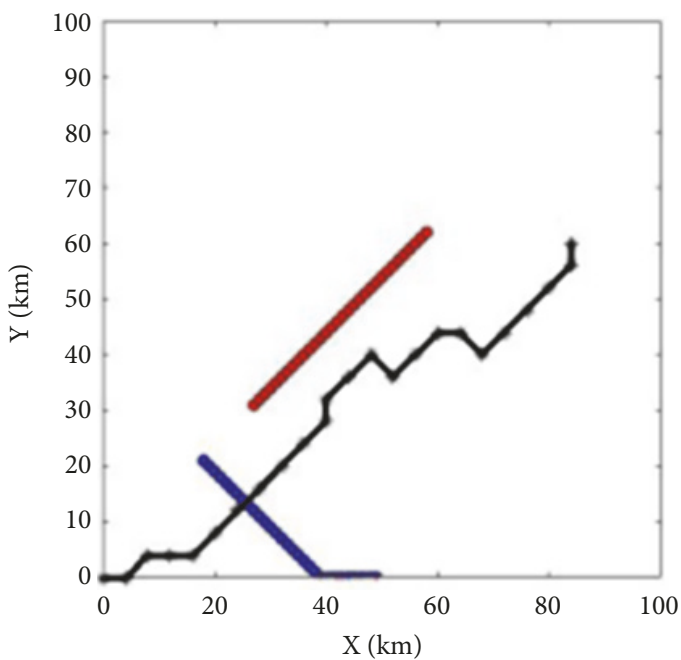

(d) $\mathrm{k}=300$

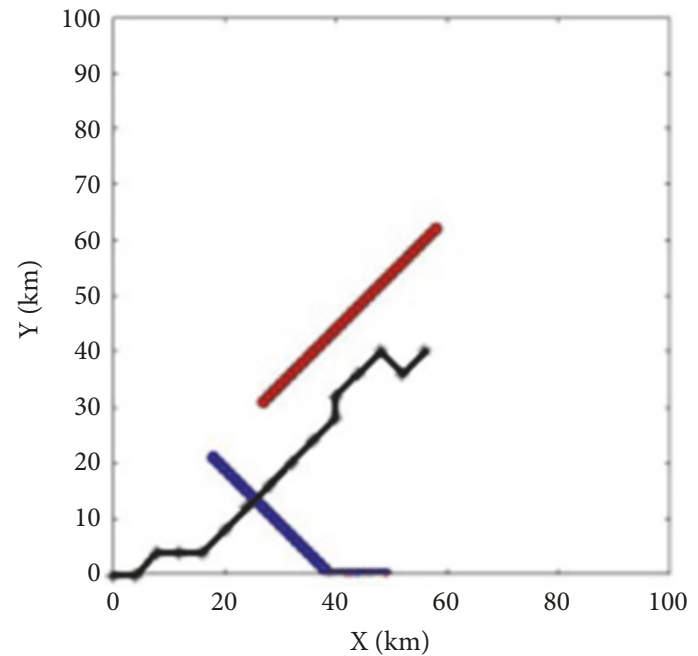

(c) $\mathrm{k}=200$

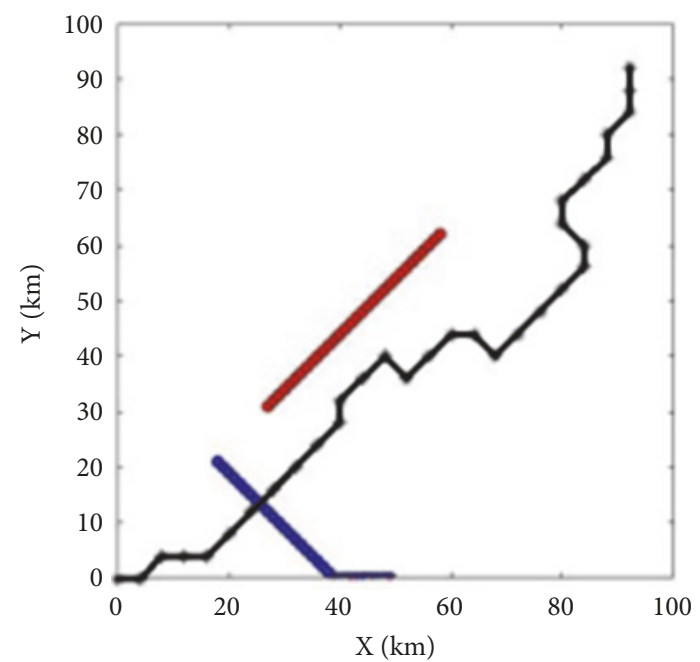

(e) $\mathrm{k}=400$

FIGURE 10: UAV path planning in one environment based on our proposed algorithm. And there are only mobile threats where (a) is an entire path and (b) to (e) are paths with iterations $=100,200,300,400$. 


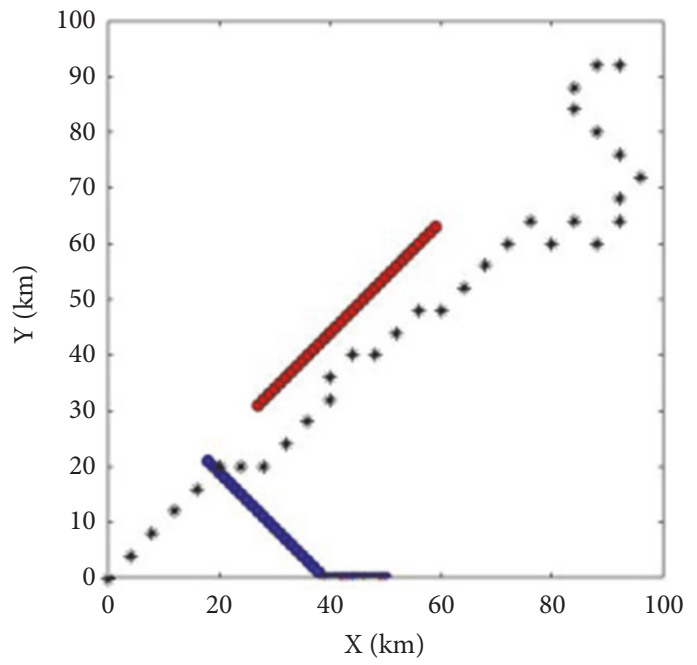

(a) Entire path

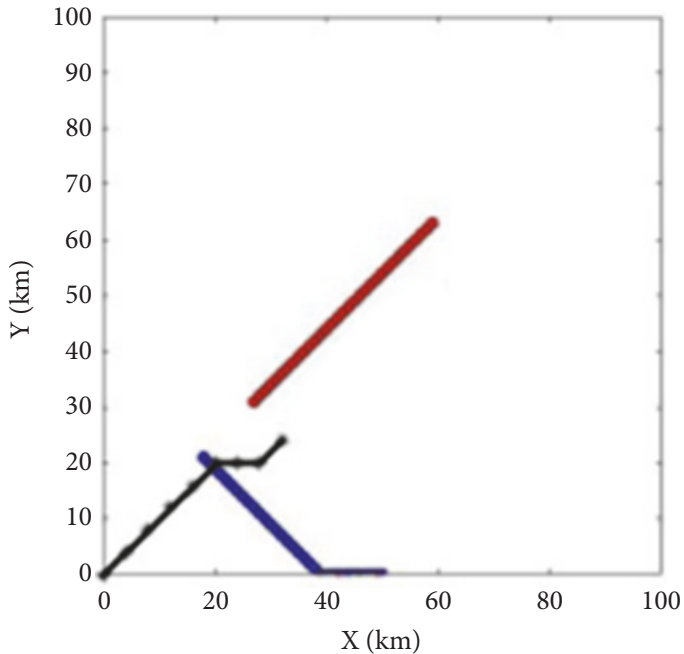

(b) $\mathrm{k}=100$

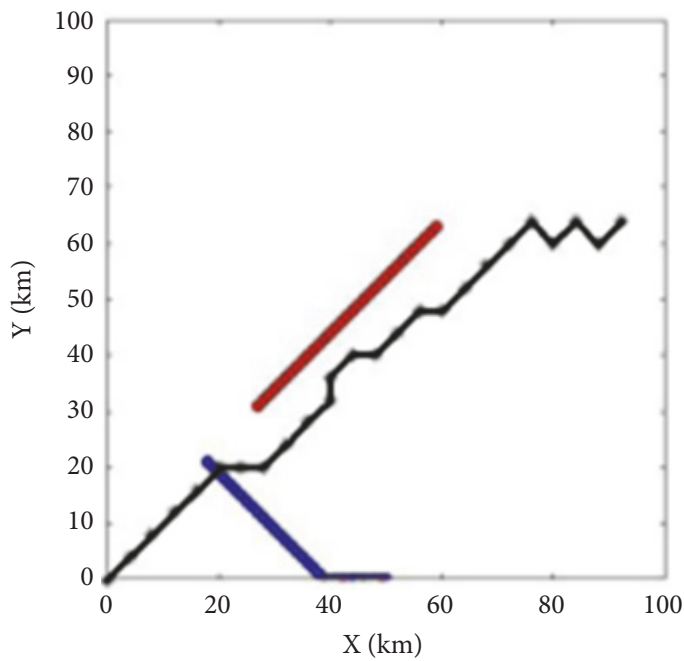

(d) $\mathrm{k}=300$

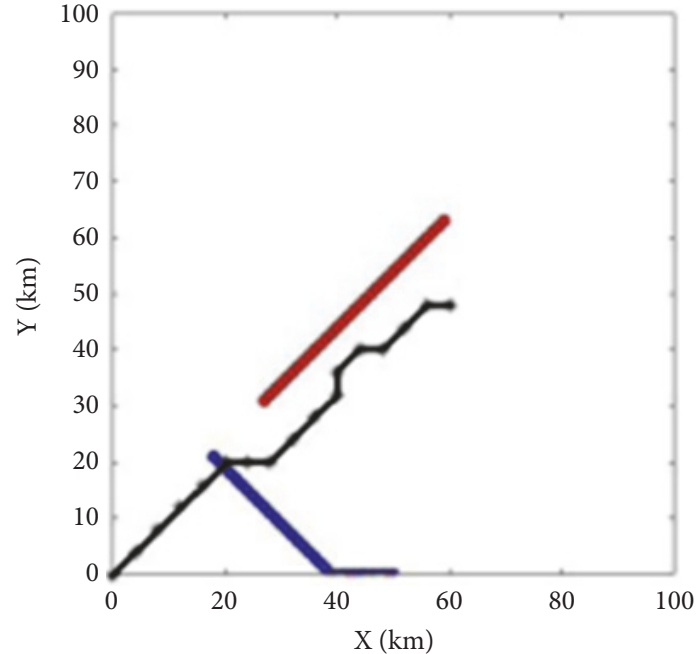

(c) $\mathrm{k}=200$

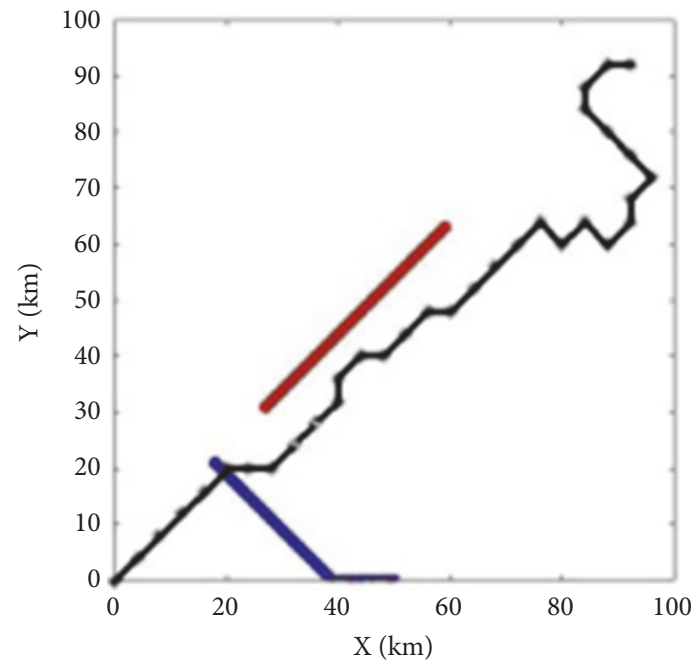

(e) $\mathrm{k}=400$

FIGURE 11: UAV path planning in one environment based on ACO algorithm. And there are only mobile threats where (a) is an entire path and (b) to (e) are paths with iterations $=100,200,300,400$. 


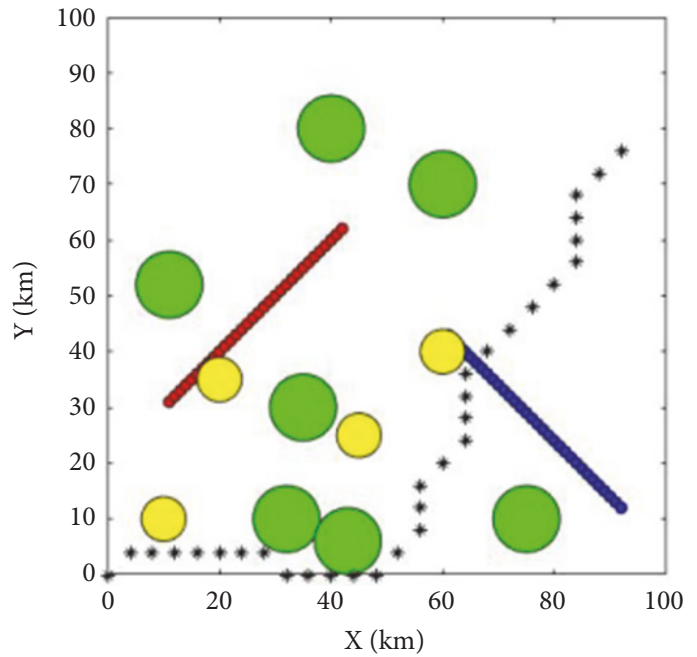

(a) Entire path

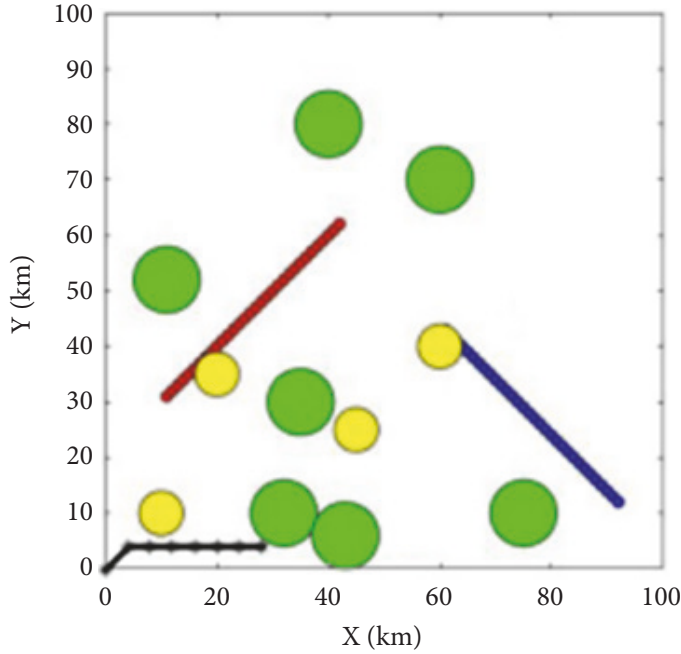

(b) $\mathrm{k}=100$

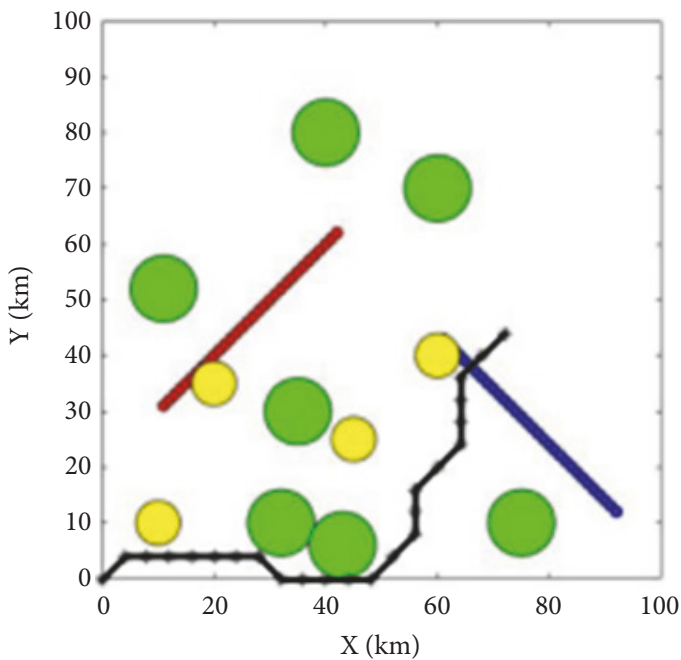

(d) $\mathrm{k}=300$

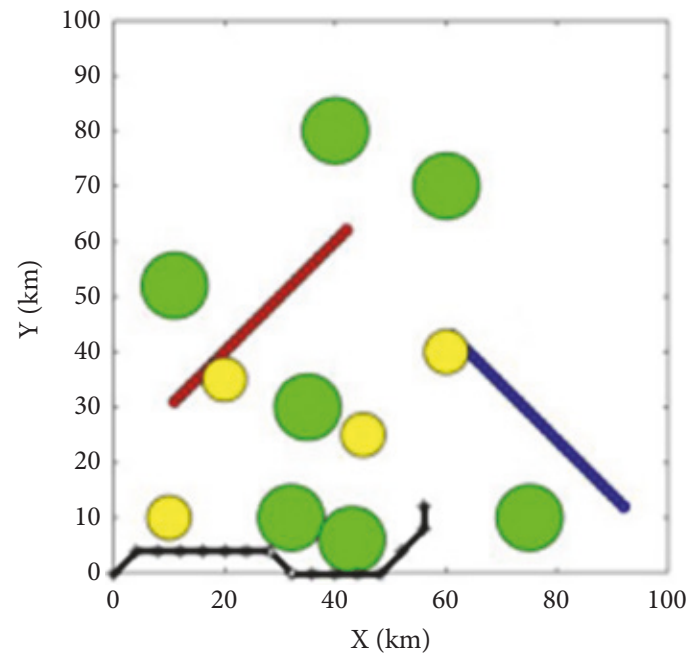

(c) $\mathrm{k}=200$

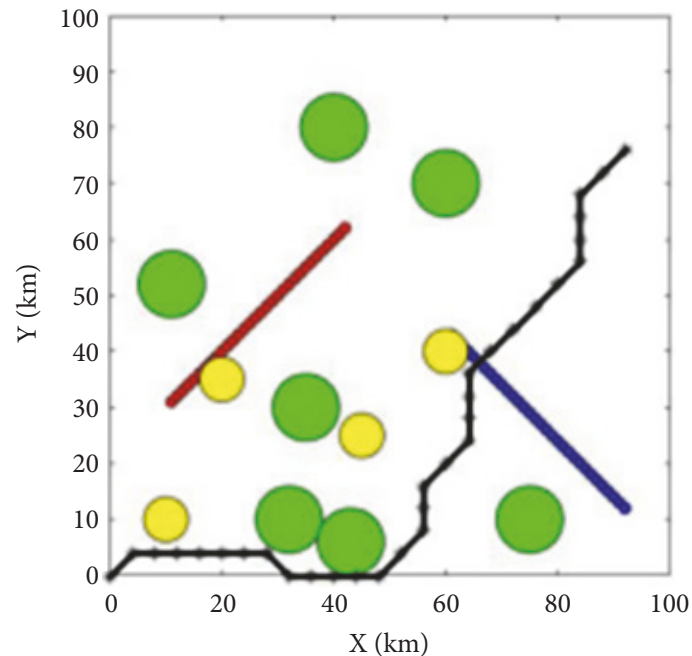

(e) $\mathrm{k}=400$

FIGURE 12: UAV path planning in another environment based on our proposed algorithm. And there are both static and mobile threats where (a) is an entire path and (b) to (e) are paths with iterations $=100,200,300,400$. 


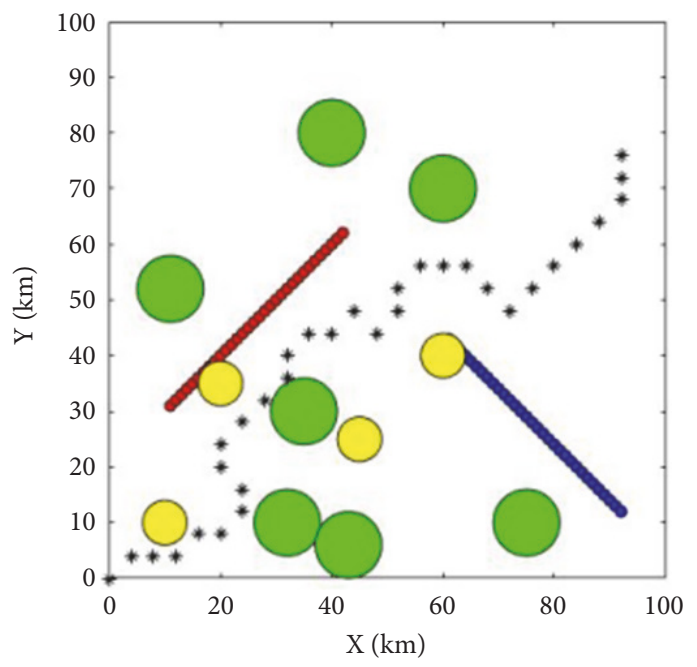

(a) Entire path

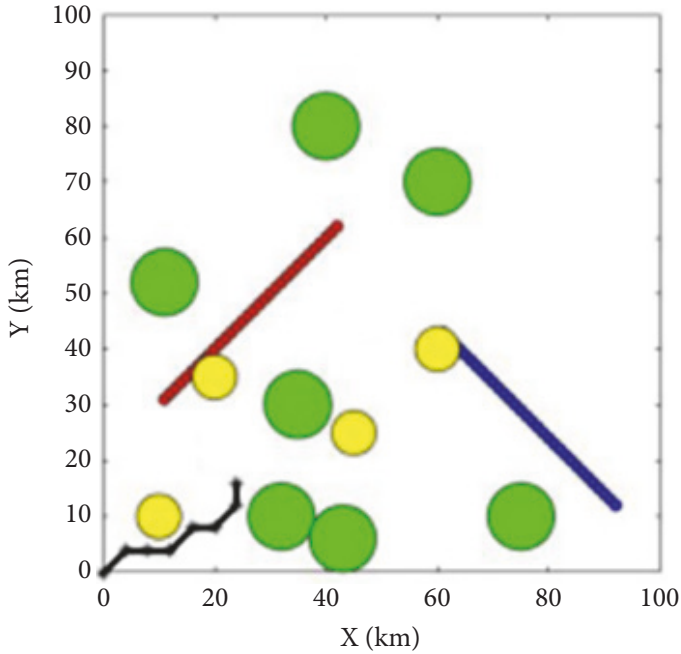

(b) $\mathrm{k}=100$

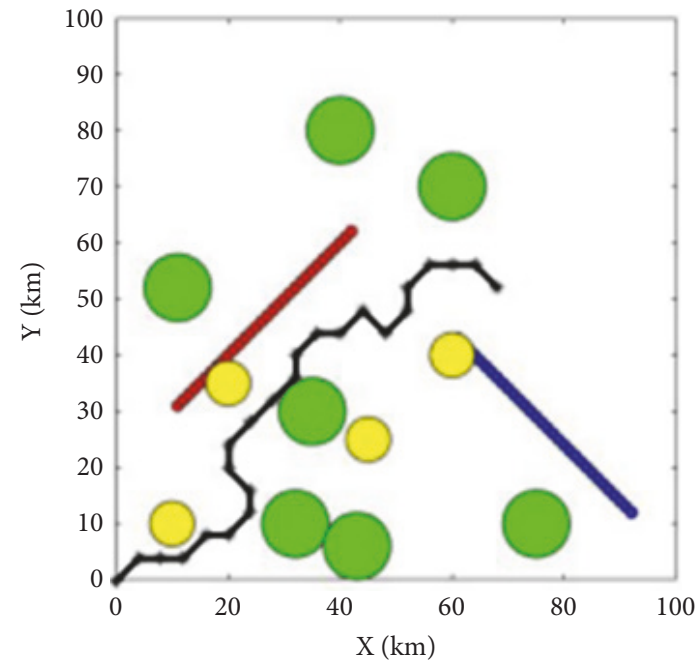

(d) $\mathrm{k}=300$

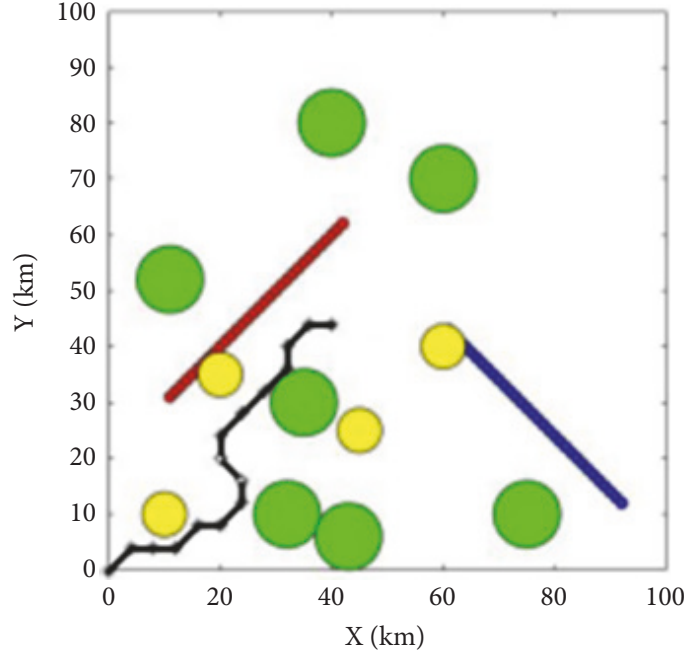

(c) $\mathrm{k}=200$

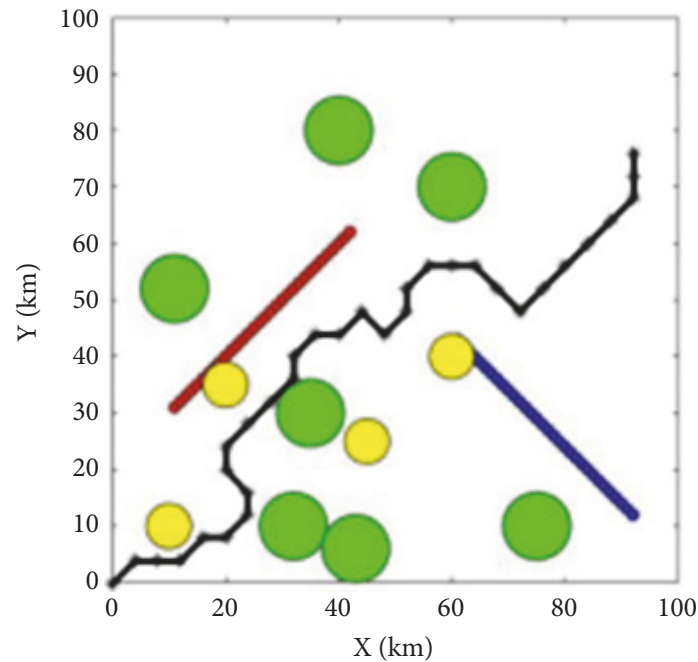

(e) $\mathrm{k}=400$

FIGURE 13: UAV path planning in another environment based on ACO algorithm. And there are both static and mobile threats where (a) is an entire path and (b) to (e) are paths with iterations $=100,200,300,400$. 


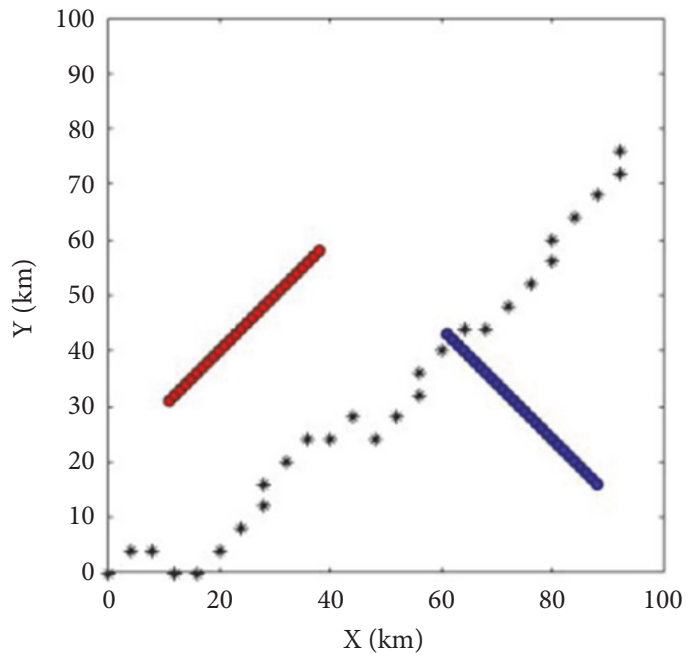

(a) Entire path

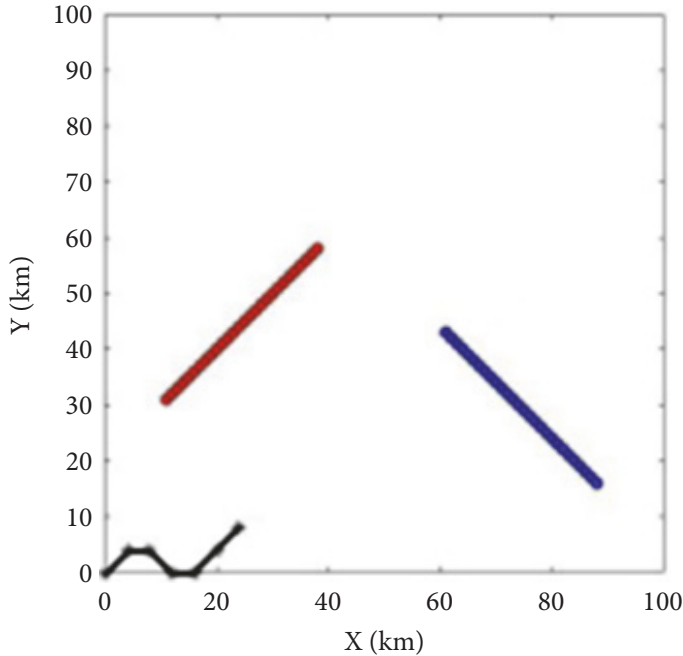

(b) $\mathrm{k}=100$

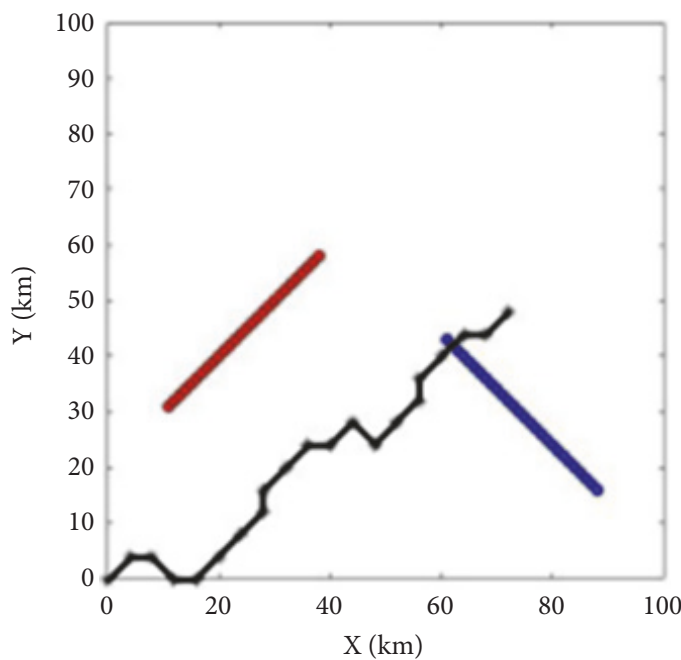

(d) $\mathrm{k}=300$

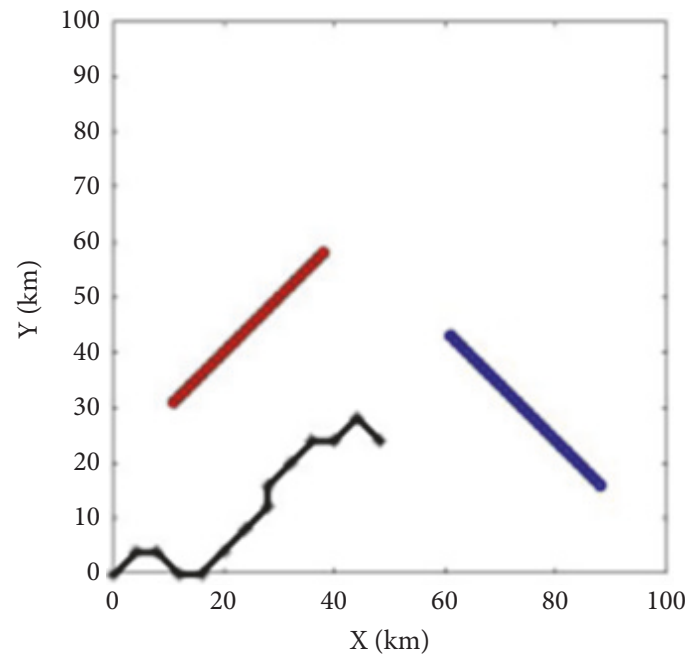

(c) $\mathrm{k}=200$

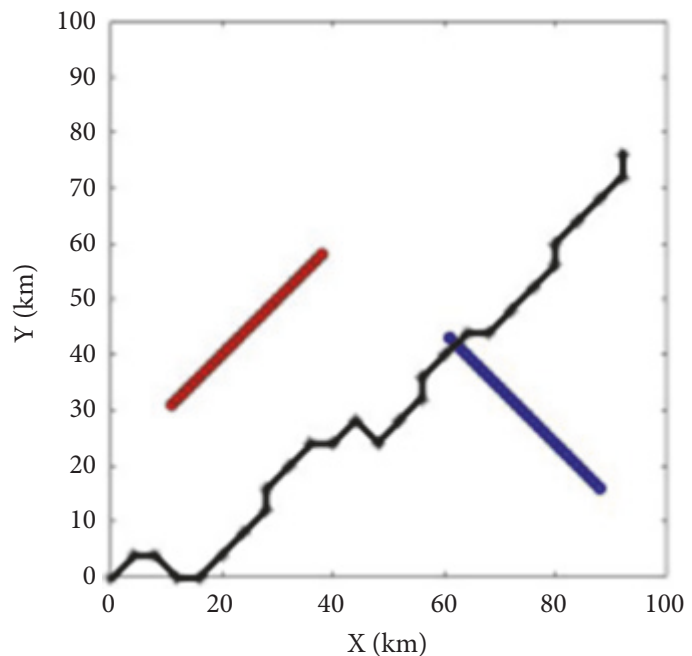

(e) $\mathrm{k}=400$

FIGURE 14: UAV path planning in another environment based on our proposed algorithm. And there are only mobile threats where (a) is an entire path and (b) to (e) are paths with iterations $=100,200,300,400$. 


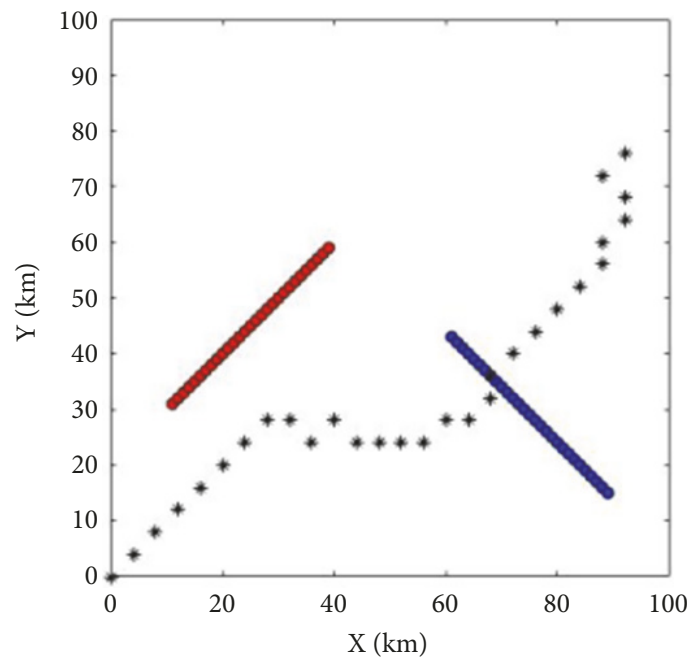

(a) Entire path

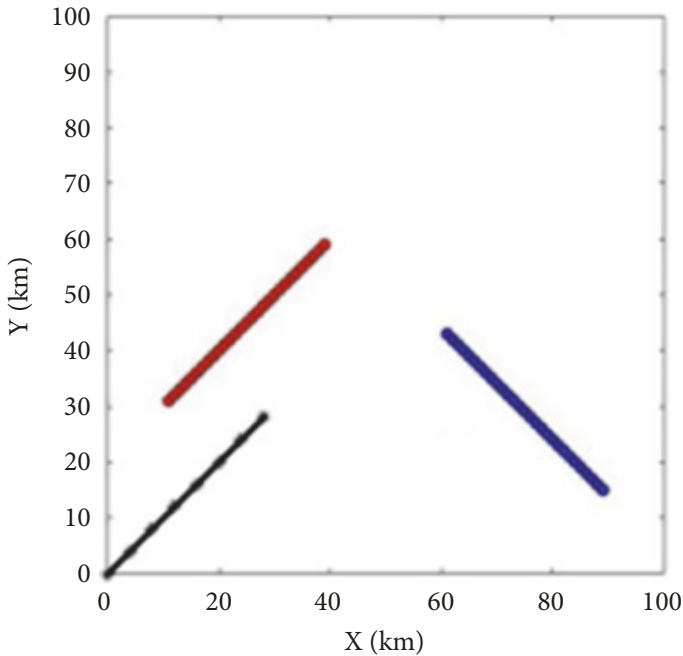

(b) $\mathrm{k}=100$

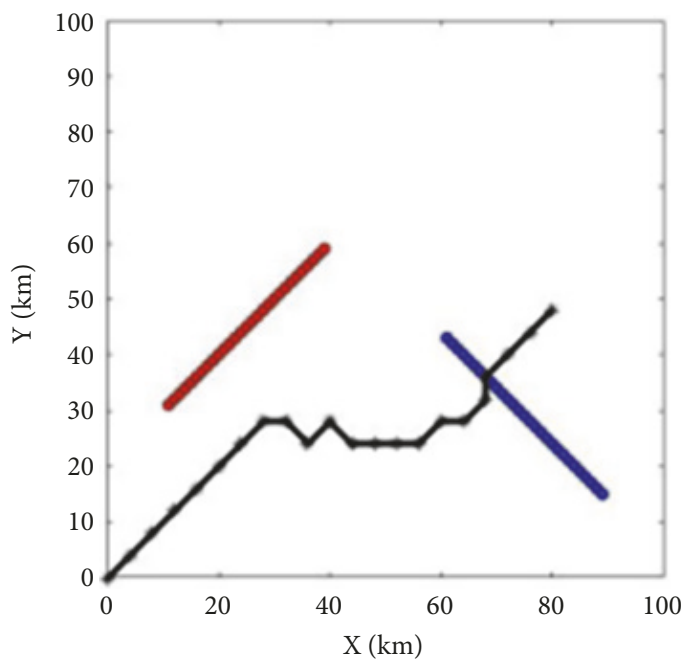

(d) $\mathrm{k}=300$

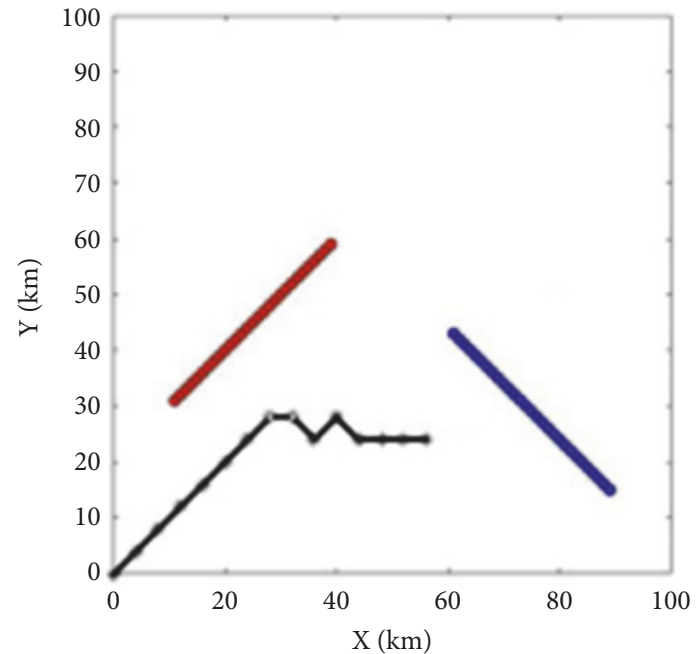

(c) $k=200$

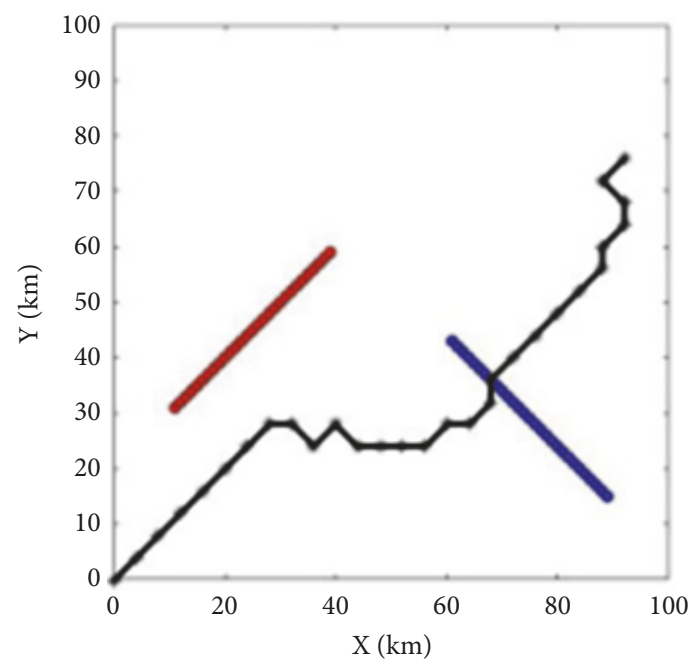

(e) $\mathrm{k}=400$

FIGURE 15: UAV path planning in another environment based on ACO algorithm. And there are only mobile threats where (a) is an entire path and (b) to (e) are paths with iterations $=100,200,300,400$. 
TABLE 1: Performance evaluations on optimal planning paths between different methods.

\begin{tabular}{|c|c|c|c|c|c|}
\hline Metric & & & Cost of mobile threats & Cost of path & Total cost \\
\hline \multirow{4}{*}{ Set 1} & \multirow{2}{*}{ Mobile and static threats } & ACO algorithm & 12.4028 & 17.6167 & 30.0194 \\
\hline & & Our algorithm & 2.8986 & 19.3137 & 22.2123 \\
\hline & \multirow{2}{*}{ Mobile threats } & ACO algorithm & 12.8763 & 16.7765 & 29.6528 \\
\hline & & Our algorithm & 12.3041 & 15.8794 & 28.1835 \\
\hline \multirow{4}{*}{ Set 2} & \multirow{2}{*}{ Mobile and static threats } & ACO algorithm & 6.9403 & 11.7823 & 18.7226 \\
\hline & & Our algorithm & 2.7300 & 13.7539 & 16.4839 \\
\hline & \multirow{2}{*}{ Mobile threats } & ACO algorithm & 3.5272 & 12.3882 & 15.9155 \\
\hline & & Our algorithm & 2.5983 & 12.3882 & 14.9865 \\
\hline \multirow{4}{*}{ Set 3} & \multirow{2}{*}{ Mobile and static threats } & ACO algorithm & 5.3881 & 15.5480 & 20.9361 \\
\hline & & Our algorithm & 2.2357 & 14.5539 & 16.7896 \\
\hline & \multirow{2}{*}{ Mobile threats } & ACO algorithm & 3.6721 & 14.5137 & 18.1858 \\
\hline & & Our algorithm & 2.3367 & 13.9480 & 16.2847 \\
\hline
\end{tabular}

Step 4 (output). Once the termination criteria are met, the iteration process stops. The way points generated during the process for each step will be linked sequentially. The way points finally form final a global optimum path.

\section{Result}

To validate the effectiveness of the proposed algorithm in solving the two-dimensional path planning problem, experiments are conducted and also comparisons of our proposed method are made with ACO with the same parameters settings.

The Figures 6 and 7 below show the relations between the length of the optimal path and the number of iterations based on our proposed method. Figures 6(a) and 7(a) show the path length with both static and mobile threats while Figures 6(b) and 7 (b) show the path length with only mobile threats. It can be seen that the path length tends to be a fixed value with the increase of iterations and the fixed value is also called the optimal solution. And compared with the path with both static and mobile threats, the path with only mobile threats has the maximum path length when the iterations are lower.

Two different kinds of mobile threats are denoted by blue and red circles, respectively, while two different kinds of static threats are denoted by green and yellow circles. Meanwhile, two different environments are included in our experiments. The former has nine static threats and two mobile threats while the latter has a total of eleven threats and two mobile threats.

Experiments for comparing the performance of the proposed algorithm and ACO are carried out. The processes of path planning are shown in Figures 8-15. Meanwhile, Figures $8,9,12$, and 13 show the planning path with both static and mobile threats while Figures 10, 11, 14, and 15 show the planning path with only mobile threats. Moreover, UAV planning paths based on our algorithm are shown in Figures $8,10,12$, and 14 . The planning paths obtained for UAVs through ACO algorithm are also shown in Figures 9, 11, 13, and 15. It is shown that, with the increase of iterations in the process of path planning, the map search of our proposed algorithm is more thorough than ACO algorithm, so the length of planning path obtained by our algorithm is usually shorter and smoother.

A set of metrics are used to measure the optimality of the proposed method, considering the ant colony optimization algorithm as a reference. The measures are the cost of mobile threats, the cost of path, and the total cost.

It can be seen in Table 1, in the environment of mobile and static threats and mobile threats, our proposed algorithm performs better than ACO algorithm in the cost of mobile threats, the cost of path, and the total cost. This demonstrates that the proposed path planning approach enables the UAVs to cost less to travel from the start point to the destination.

\section{Conclusion}

In this study, both mobile threats and static threats are considered to simulate the actual situations better. A series of experiments demonstrate that the proposed algorithm can effectively plan a threats free path. We focus on the problem of finding a planning path with the lowest cost for UAVs. A coordinate system transformation is used for simplicity to reduce the computational complexity. Meanwhile, the cost value is considered as a scalar value of mobile threats which are vectors actually. In this way, the moving direction of $\mathrm{UAV}$ is more optimal and the searching process for optimal planning path is more efficient. Moreover, compared with ACO, the planning paths obtained based on our algorithm are smoother.

For the proposed algorithm, it can be seen from the results that the planning path may just go over with obstacles. However, the actual UAV can not be abstracted as a particle because of its size so that flying in this path is not safe. In the future work, a data preprocessing needs to be taken to leave a certain security space. And in our model, the radius and moving direction of a mobile threat are constant which may change in fact. In this way, we should also set changeable radius and moving directions for mobile threats in the environment to simulate reality scene. Moreover, more algorithms for UAV path planning such as standard PSO, FA, and GA should be taken into account in experiments compared with the algorithm we put forward. And the path 
planning in two-dimensional environment can be converted into a three-dimensional environment to achieve a more precious path planning.

\section{Data Availability}

The data used to support the findings of this study are available from the corresponding author upon request.

\section{Conflicts of Interest}

The authors declare that they have no conflicts of interest.

\section{References}

[1] Y. G. Fu, M. Y. Ding, C. P. Zhou, and H. P. Hu, "Route planning for unmanned aerial vehicle (UAV) on the sea using hybrid differential evolution and quantum-behaved particle swarm optimization," IEEE Transactions on Systems, Man, and Cybernetics: Systems, vol. 43, no. 6, pp. 1451-1465, 2013.

[2] R. R. Pitre, X. R. Li, and R. Delbalzo, "UAV route planning for joint search and track missionsan information-value approach," IEEE Transactions on Aerospace and Electronic Systems, vol. 48, no. 3, pp. 2551-2565, 2012.

[3] M. Kothari and I. Postlethwaite, "A probabilistically robust path planning algorithm for UAVs using rapidly-exploring random trees," Journal of Intelligent \& Robotic Systems, vol. 71, no. 2, pp. 231-253, 2013.

[4] S. Wei, G. Yan, and H. Yu, "Path planning for UAV based on mixed ant colony algorithm," Journal of System Simulation, vol. 23, no. 3, pp. 574-576, 2011.

[5] X. Peng and D. Xu, "Intelligent Online Path Planning for UAVs in Adversarial Environments," International Journal of Advanced Robotic Systems, vol. 9, no. 1, p. 3, 2012.

[6] A. Melingui, R. Merzouki, J. B. Mbede, and T. Chettibi, "A novel approach to integrate artificial potential field and fuzzy logic into a common framework for robots autonomous navigation," Proceedings of the Institution of Mechanical Engineers, Part I: Journal of Systems and Control Engineering, vol. 228, no. 10, pp. 787-801, 2014.

[7] H. Duan, Y. Zhang, and S. Liu, "Multiple UAVs/UGVs heterogeneous coordinated technique based on Receding Horizon Control (RHC) and velocity vector control," Science China Technological Sciences, vol. 54, no. 4, pp. 869-876, 2011.

[8] S. Zhai and T. Jiang, "A new sense-through-foliage target recognition method based on hybrid differential evolution and selfadaptive particle swarm optimization-based support vector machine," Neurocomputing, vol. 149, pp. 573-584, 2015.

[9] B. Song, Z. Wang, and L. Zou, "On Global Smooth Path Planning for Mobile Robots using a Novel Multimodal Delayed PSO Algorithm," Cognitive Computation, vol. 9, no. 1, pp. 5-17, 2017.

[10] E. G. Tsardoulias, A. Iliakopoulou, A. Kargakos, and L. Petrou, "A Review of Global Path Planning Methods for Occupancy Grid Maps Regardless of Obstacle Density,' Journal of Intelligent \& Robotic Systems, vol. 84, no. 1-4, pp. 829-858, 2016.

[11] Y. Liu, X. Zhang, X. Guan, and D. Delahaye, "Potential Odor Intensity Grid Based UAV Path Planning Algorithm with Particle Swarm Optimization Approach," Mathematical Problems in Engineering, vol. 2016, 2016.

[12] A. Askari, M. Mortazavi, H. A. Talebi, and A. Motamedi, "A new approach in UAV path planning using Bezier-Dubins continuous curvature path," Proceedings of the Institution of Mechanical Engineers, Part G: Journal of Aerospace Engineering, vol. 230, no. 6, pp. 1103-1113, 2015.

[13] M. Yao and M. Zhao, "Unmanned aerial vehicle dynamic path planning in an uncertain environment," Robotica, vol. 33, no. 3, pp. 611-621, 2015.

[14] B. Kane T and D. Theory, "Real-Time Obstacle Avoidance for Manipulators and Mobile Robot," The International Journal of Robotics Research, vol. 5, no. 1, 1986.

[15] M. G. Park and M. C. Lee, "A new technique to escape local minimum in artificial potential field based path planning," KSME International Journal, vol. 17, no. 12, pp. 1876-1885, 2003.

[16] Z. Wang, J. Li, M. Fang, and Y. Li, "A Multimetric Ant Colony Optimization Algorithm for Dynamic Path Planning in Vehicular Networks," International Journal of Distributed Sensor Networks, vol. 2015, 2015.

[17] S. Asghari and K. Azadi, "A reliable path between target users and clients in social networks using an inverted ant colony optimization algorithm," Karbala International Journal of Modern Science, vol. 3, no. 3, pp. 143-152, 2017.

[18] Z. Nagórny, "Application of a Modified Hopfield Network to the Traveling Salesman Problem," Economics and Organization of Enterprise, vol. 6, no. 4, pp. 58-66, 2009.

[19] M. Gu, "The application of the fuzzy ART neural network algorithm," Applied Mechanics and Materials, vol. 513-517, pp. 1783-1786, 2014.

[20] N. Zeng, H. Zhang, Y. Chen, B. Chen, and Y. Liu, "Path planning for intelligent robot based on switching local evolutionary PSO algorithm," Assembly Automation, vol. 36, no. 2, pp. 120-126, 2016.

[21] N. Zeng, Z. Wang, H. Zhang, and F. E. Alsaadi, "A Novel Switching Delayed PSO Algorithm for Estimating Unknown Parameters of Lateral Flow Immunoassay," Cognitive Computation, vol. 8, no. 2, pp. 143-152, 2016.

[22] N. Zeng, H. Zhang, W. Liu, J. Liang, and F. E. Alsaadi, "A switching delayed PSO optimized extreme learning machine for short-term load forecasting," Neurocomputing, vol. 240, pp. 175182, 2017.

[23] J. H. Holland, Adaptation in Natural and Artificial Systems, University of Michigan Press, Ann Arbor, Michigan, USA, 2nd edition, 1975.

[24] X. Yuan, M. Elhoseny, H. K. El-Minir, and A. M. Riad, "A Genetic Algorithm-Based, Dynamic Clustering Method Towards Improved WSN Longevity," Journal of Network and Systems Management, vol. 25, no. 1, pp. 21-46, 2017.

[25] R. J. Szczerba, P. Galkowski, I. S. Glickstein, and N. Ternullo, "Robust algorithm for real-time route planning," IEEE Transactions on Aerospace and Electronic Systems, vol. 36, no. 3, pp. 869-878, 2000.

[26] P. Melchior, B. Orsoni, O. Lavialle, A. Poty, and A. Oustaloup, "Consideration of obstacle danger level in path planning using $\mathrm{A} *$ and fast-marching optimisation: comparative study," Signal Processing, vol. 83, no. 11, pp. 2387-2396, 2003. 


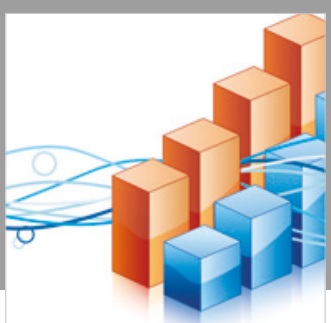

Advances in

Operations Research

\section{-n-m}
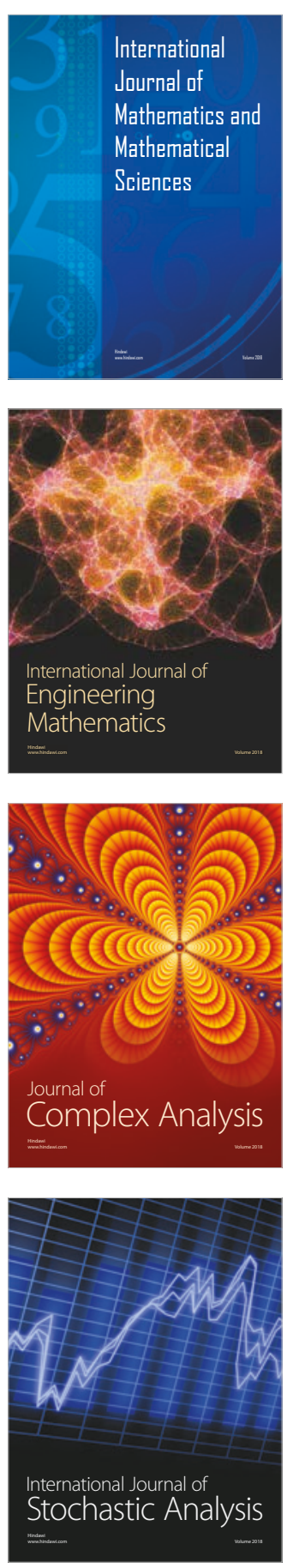
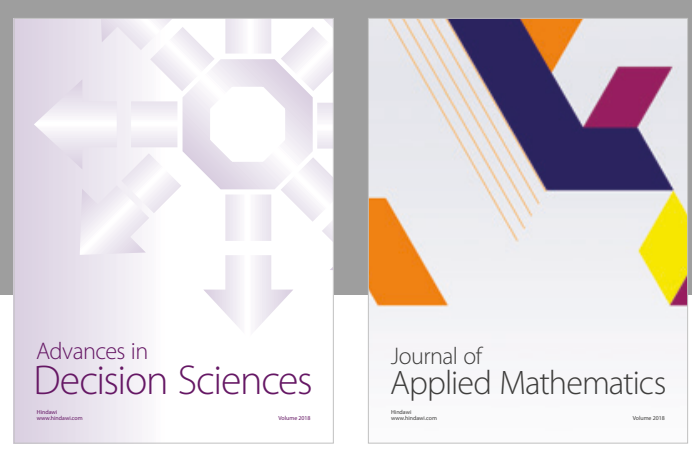

Journal of

Applied Mathematics
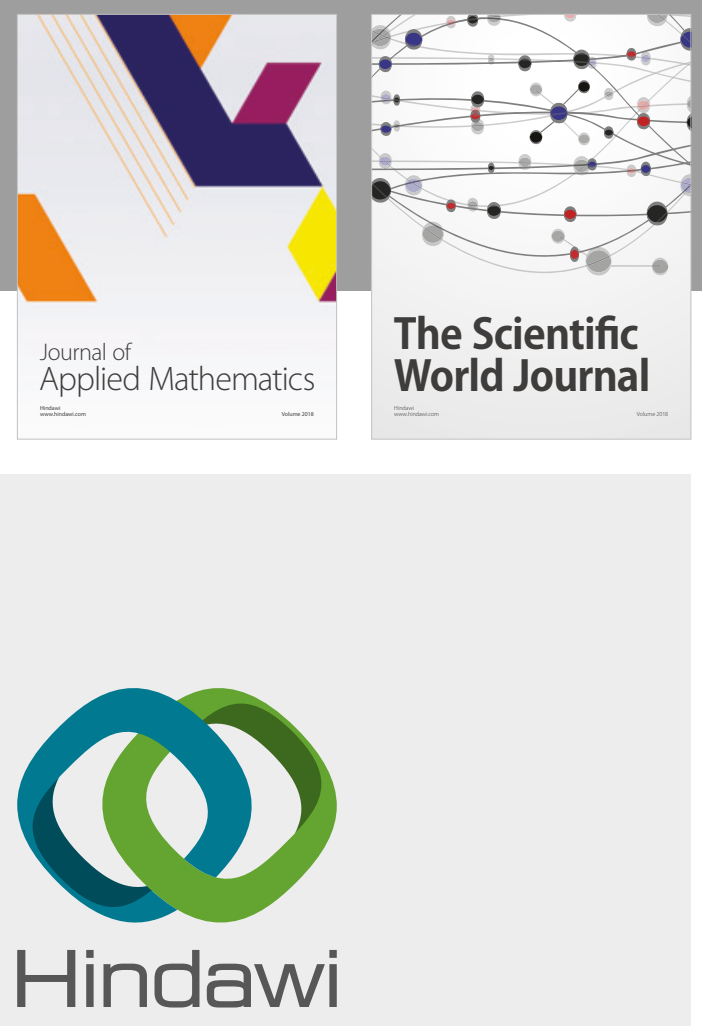

Submit your manuscripts at

www.hindawi.com

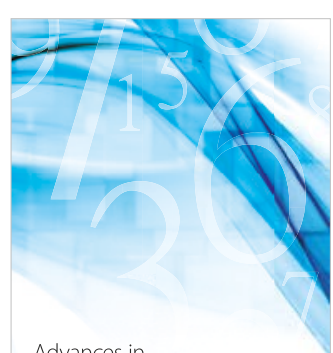

Advances in
Numerical Analysis
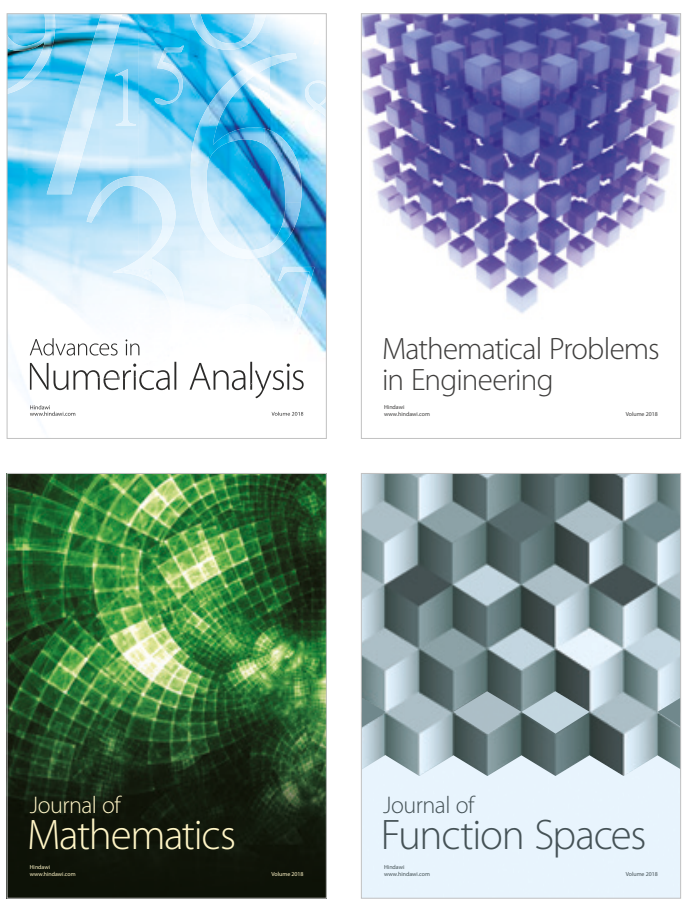

Mathematical Problems in Engineering

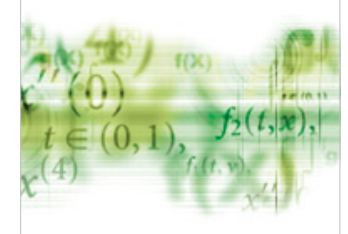

International Journal of

Differential Equations

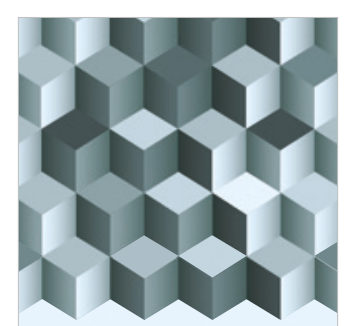

Journal of

Function Spaces
The Scientific

World Journal

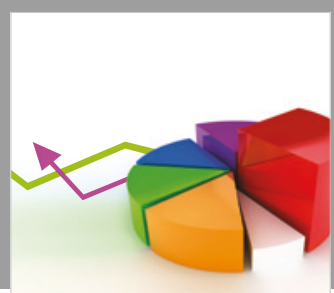

Journal of

Probability and Statistics
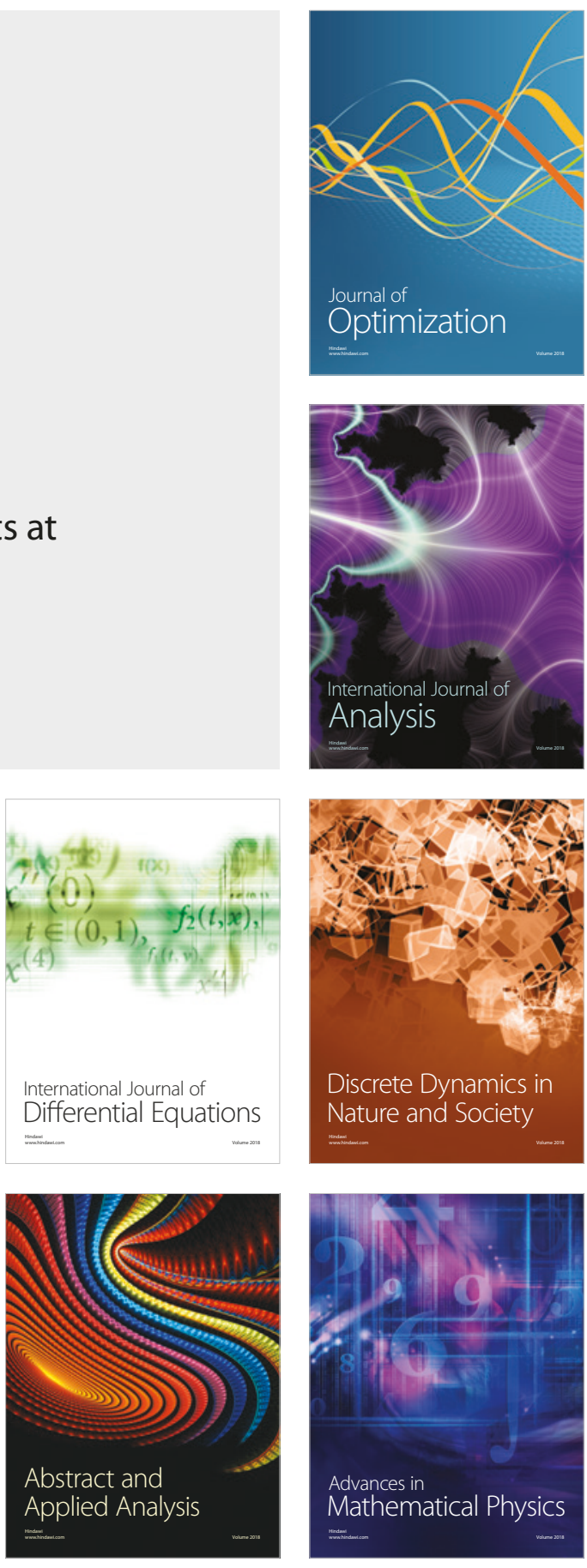